\title{
Numerical investigations on the flow over cuboids with different aspect ratios and the emitted noise
}

\author{
Yanan Wang (王亚南), ${ }^{1}$ a) David Thompson, ${ }^{1,}$ b) and Zhiwei Hu (胡志伟) ${ }^{2}$, c) \\ ${ }^{1)}$ Institute of Sound and Vibration Research, University of Southampton, Southampton, SO17 1BJ, \\ United Kingdom. \\ ${ }^{2)}$ Department of Aeronautical and Astronautical Engineering, University of Southampton, Southampton, SO17 1BJ, \\ United Kingdom.
}

(Dated: 4 March 2020)

The effect of aspect ratio on the flow over cuboids in cross flow is investigated numerically, together with the noise emitted to the far field. The near-field flow features are obtained using the delayed detached eddy simulation approach and the far-field noise is predicted using the Ffowcs Williams-Hawkings acoustic analogy. Ten values of aspect ratio are studied in the current work ranging from 1/6 to 12 at three Reynolds numbers between 50,000 and 200,000. Aerodynamic coefficients, flow patterns and pressure distributions along the cuboid surfaces are studied in detail with respect to cuboid aspect ratio for different Reynolds numbers. It is found that the separated flow from the leading edge reattaches to the lateral surfaces of cuboids with aspect ratios smaller than unity, while a secondary vortex is formed near the trailing edge for larger aspect ratios. In addition, the aspect ratio has considerable effect on the tonal peak in the far-field noise spectra from the cuboids occurring for aspect ratios greater than 1.

Keywords: Aspect ratio; Flow patterns; Aerodynamic noise

\section{INTRODUCTION}

The flow around cuboids or finite length square cylinders differs considerably from that for an infinite square

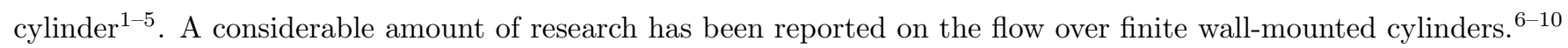
A detailed review about the flow structures in the wake of wall-mounted cylinders and the radiated noise was presented by Porteous et al ${ }^{11}$ Different models were summarised describing the relationship between the shed vortices from the free end and the base of the cylinders. Measurements on the flow-induced noise from wall-mounted cylinders were also reviewed and compared. More recently, Yauwenas et al ${ }^{[12}$ experimentally and numerically investigated the effect of aspect ratio on the flow structures of square finite wall-mounted cylinders. The wake velocity and surface pressure of the cylinders were measured in wind tunnels using hot-wire anemometers and pressure taps respectively. The aspect ratios studied experimentally ranged from 1.4 to 21.4 at Reynolds numbers of $1.1 \times 10^{4}$ and $1.4 \times 10^{4}$ based on the cylinder width. Complementary simulations were also performed using large eddy simulation (LES) to obtain more

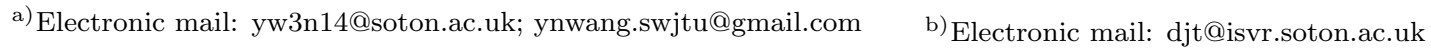

c) Electronic mail: z.hu@soton.ac.uk
} 
flow features for four representative aspect ratios. A parametric diagram was proposed to categorise the transition of wake structures taking into account both the boundary layer thickness and the aspect ratio.

Zhang et al ${ }^{13}$ performed direct numerical simulation (DNS) on a wall-mounted cylinder with an aspect ratio of 4 for Reynolds numbers between 50 and 1000 based on the cylinder width. Three types of mean streamwise vortices around the cylinder were categorised in detail. The effect of the incoming boundary layer thickness on the shedding modes of a wall-mounted cylinder with an aspect ratio of 7 was investigated by Behera and Saha ${ }^{14}$ employing DNS. Six incoming turbulent boundary layer thicknesses were considered at a Reynolds number of 250 based on the cylinder width. Both anti-symmetric and symmetric shedding patterns were observed in the wake of the cylinder. The symmetric shedding mode occurred more frequently as the boundary layer thickness was increased. Cao et al. 15 employed the Immersed Boundary Method to investigate surface flow patterns and pressure on wall-mounted cylinders with aspect ratios of 3 and 4 . The Reynolds number based on the cylinder width was $5 \times 10^{4}$ and two angles of attack $\left(0^{\circ}\right.$ and $\left.15^{\circ}\right)$ were considered. Near-wall flow topologies were analysed to clarify the three-dimensional effect of the flow over the finite length square cylinders, including critical points $\sqrt{16}$ of wall streamlines, lines of flow separation and reattachment. In addition, Beitel et al.$^{17}$ measured the aerodynamic forces and the bending moment of circular wall-mounted cylinders in a wind tunnel. The aspect ratios ranged from 0.5 to 11 and the Reynolds number was $6.5 \times 10^{4}$ based on the diameter of the cross-section. Glumac et al $\frac{18}{18}$ contributed a database of the flow above the roof of a tall building, represented by a wall-mounted square cylinder with an aspect ratio of 3 in a wind tunnel. The principal building was surrounded by four identical buildings and the Reynolds number was $1.4 \times 10^{5}$ based on the side length of the roof. Velocities above the roof of the principal building were measured using hot-wire anemometry; the surface pressure distribution was obtained by 64 pressure taps.

Moreau and Doolan measured the noise from wall-mounted cylinders with finite length in an anechoic wind tunnel!19 Both circular and square cross-sections were considered. The aspect ratio ranged between 1.6 and 22.6 for the circular cylinders and between 0.97 and 13.6 for the square cylinders. Three flow speeds were taken into account with corresponding Reynolds numbers between $1.0 \times 10^{4}$ and $1.4 \times 10^{4}$ for the circular cylinder based on its diameter and between $1.6 \times 10^{4}$ and $2.4 \times 10^{4}$ for the square cylinders based on the cross-section width. Multiple tonal peaks were found in the noise spectra, which were related to different vortex cells formed in the wake. The number and strength of these vortex cells were affected by the aspect ratio. Porteous et al 20 conducted acoustic measurements on wall-mounted square cylinders with aspect ratios ranging between 0.29 and 22.9 in an anechoic wind tunnel. The Reynolds number was $1.4 \times 10^{4}$ based on the width of the cylinder. Based on the number of tonal peaks observed, four shedding regimes were categorised. Velocity profiles in the wake of these cylinders were also measured using hot-wire anemometers. A novel phase eduction approach was adopted to visualise the looping vortices in the wake, spatially mapping points where the velocity was in phase with the far-field acoustic pressure measured by a microphone. Becker et al. 21 measured the sound from a wall-mounted square cylinder with an aspect ratio of 6 and also the mean flow field using laser Doppler anemometry. A tonal peak at a Strouhal number $S t=0.1$ was found for the finite-length square cylinder, which was lower than $S t=0.13$ for square cylinders with infinite length. ${ }^{22}$ The noise emitted from

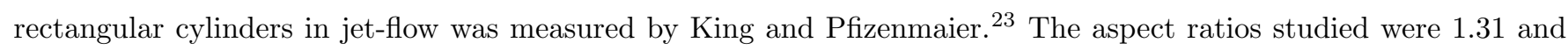
2.2. In their work, it was believed that the measured sound was only from the cylinder, because the fixture, where the cylinder was mounted, was out of the nozzle region.

As reviewed above, most of the previous studies were on the flow structures or the noise of wall-mounted square 
cylinders with different aspect ratios. The effect of the aspect ratio on the aerodynamic and the aeroacoustic features of square cylinders in cross flow has not been investigated to the same extent, although this is also useful for many engineering applications. In the current work, the near-field flow patterns and the far-field noise are characterized both quantitatively and qualitatively for cuboids in cross flow with aspect ratios ranging from $1 / 6$ to 12 . Three Reynolds numbers are considered between 50,000 and 200,000. Firstly, details of the flow features are obtained using the delayed detached eddy simulation (DDES) method in the open source software OpenFOAM, including the fluctuating pressure on the cuboid surfaces responsible for the dipole noise sources. Subsequently, the far-field noise is predicted based on the surface fluctuating pressure, using the Ffowcs Williams-Hawkings (FW-H) acoustic analogy in the commercial software FLUENT. In this way, the database of this topic is extended for a better understanding of the underlying physics.

\section{NUMERICAL METHOD}

\section{A. Governing equations}

Since the maximum Mach number studied in this work is about 0.12 , the flow is assumed to be incompressible; it is generally considered that this is a reasonable assumption for Mach numbers up to about $0.32^{24}$ For incompressible flow, the flow density $\rho$ is considered as constant ${ }^{25}$ and the governing Navier-Stokes (N-S) equations are simplified as follows (in tensor notation):

$$
\begin{array}{r}
\frac{\partial u_{j}}{\partial x_{j}}=0 \\
\frac{\partial u_{i}}{\partial t}+u_{j} \frac{\partial u_{i}}{\partial x_{j}}=-\frac{1}{\rho} \frac{\partial p}{\partial x_{i}}+\nu \frac{\partial^{2} u_{i}}{\partial x_{j} \partial x_{j}}
\end{array}
$$

where $u_{i}$ and $u_{j}$ are velocity components along the coordinate directions $x_{i}$ and $x_{j}$, while $i$ or $j$ equals $1,2,3$, representing the streamwise, cross-flow and spanwise directions respectively. $t$ is the time, $p$ is the pressure and $\nu$ is the kinematic viscosity.

To solve the governing equations numerically, different turbulence models have been proposed such as Reynoldsaveraged Navier-Stokes (RANS), LES and DNS. The fidelity level of these approaches increases sequentially, but so do the mesh requirement and computational cost. Only time-averaged flow features are captured by RANS, while the fluctuating components are modelled by the Reynolds-stress tensor. ${ }^{26}$ Therefore, for flows with massive separation and numerous unsteadiness in the wake, RANS models become limited in their ability to capture the flow characteristics. LES can directly resolve large eddies in the flow, which contain most of the energy; small eddies, whose behaviour tends to be universal, $\stackrel{27}{27}$ are filtered spatially and modelled by the Subgrid-Scale (SGS) models $\stackrel{28,30}{[30}$ However, for wallresolved LES at high Reynolds numbers, the required computational cost is impractically high. DNS ${ }^{31}$ can resolve the smallest Kolmogorov scale, responsible for flow dissipation, which implies even higher computational demand than LES and is impractical for applications at Reynolds numbers of industrial relevance.

The detached eddy simulation (DES) is a hybrid method designed to use RANS within the boundary layer near solid surfaces but changing to LES outside it $\sqrt[32 \sqrt{33}]{ }$ In this way, the grid resolution within the boundary layer required 
by DES is much less demanding than that of LES, which requires smaller cell aspect ratios than RANS. This hybrid method is most practical for flow with massive separation at high Reynolds numbers, balancing the flow accuracy and the computational endeavour. However, some inherent flaws, such as Modelled Stress Depletion 34 and Grid Induced Separation,, 35 may occur due to the early transition from the RANS mode to the LES mode inside the boundary layer with the refinement of the grid. To ensure the DES can treat the whole boundary layer employing the RANS mode as designed, independently of the grid resolution, an adaptive model DDES was proposed by Spalart et al! 36 More recently, the improved delayed detached eddy simulation (IDDES) was developed by Shur et al. to include an additional wall-modelled LES (WMLES) branch to the DDES ${ }^{37}$ The embedded WMLES capability in IDDES is intended to address the problem of $\log$ layer mismatch (LLM) ${ }^{37}$ which occurs with DDES when the incoming flow contains turbulent content. In this work, the studied Reynolds numbers are up to 200,000 and the incoming flow is uniform without turbulent contents, therefore, the DDES model is adopted to obtain the flow features.

The one-equation Spalart-Allmaras (S-A) model is used for the RANS mode in this work, which is capable of reproducing the viscous sublayer close to the wall $! 38$ The equation of the S-A model is as follows:

$$
\frac{\partial \tilde{\nu}}{\partial t}+u_{j} \frac{\partial \tilde{\nu}}{x_{j}}=c_{b_{1}} \tilde{S} \tilde{\nu}+\frac{1}{\sigma}\left[\frac{\partial}{\partial x_{j}}\left((\nu+\tilde{\nu}) \frac{\partial \tilde{\nu}}{\partial x_{j}}\right)+c_{b_{2}} \frac{\partial \tilde{\nu}}{\partial x_{i}} \frac{\partial \tilde{\nu}}{\partial x_{i}}\right]-c_{w_{1}} f_{w}\left(\frac{\tilde{\nu}}{d}\right)^{2}
$$

where $\nu$ is the molecular viscosity, $\tilde{\nu}$ is the modified turbulent viscosity and $d$ is the wall distance. More details can be found in the literature 32 . To include the grid size in the governing equation, the physical wall distance $d$ is replaced by $\tilde{d}$ :

$$
\tilde{d}=\min \left(d, C_{D E S} \Delta\right)
$$

where $\Delta$ is the grid size and $C_{D E S}=0.65$ is a constant ${ }^{39}$ In this way, the S-A RANS model can be transformed into a SGS model related to the mesh size for LES. Eq. (4) realises the transition process from RANS to LES within DES. To overcome the inherent deficiencies in DES mentioned above, another function $f_{d}$ is built into Eq. (4) to form the DDES. The modified equation becomes:

$$
\tilde{d}=d-f_{d} \max \left(0, d-C_{D E S} \Delta\right)
$$

with

$$
f_{d}=1-\tanh \left(\left[8 r_{d}\right]^{3}\right)
$$

and

$$
r_{d}=\frac{\nu_{t}+\nu}{\sqrt{U_{i, j} U_{i, j}} k^{2} d^{2}}
$$

where $U_{i, j}$ is the gradient of velocity, $k$ is the Karman constant. Detailed explanations are presented in reference [36]. It implies the RANS mode is used when $f_{d}$ tends to 0 , while the LES mode is used for $f_{d}=1$. 


\section{B. Ffowcs Williams-Hawkings acoustic analogy}

To obtain far-field acoustic waves, instead of solving the unsteady compressible N-S equations in a very large

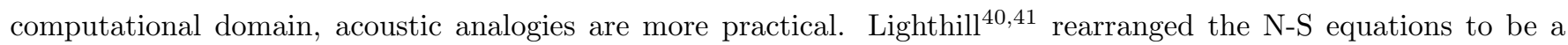
combination of a classical wave operator on the left and the equivalent noise sources on the right-hand side. Curle 42 extended the Lighthill's analogy to take into account the presence of a solid boundary in the flow. Further extension

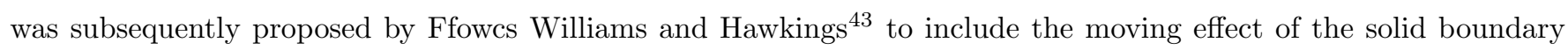
on the noise generation. The FW-H equation can be written as follows, with the wave operator on the left-hand side and the equivalent sources on the right-hand side:

$$
\frac{1}{c_{0}^{2}} \frac{\partial^{2}\left[p^{\prime} H(f)\right]}{\partial t^{2}}-\nabla^{2}\left[p^{\prime} H(f)\right]=\frac{\partial^{2}}{\partial x_{i} \partial x_{j}}\left[H(f) T_{i j}\right]-\frac{\partial}{\partial x_{i}}\left[\delta(f) F_{i}\right]+\frac{\partial}{\partial t}[\delta(f) Q]
$$

In Equation (8), $c_{0}$ is the sound speed, $H(f)$ is the Heaviside function while $f$ is used to indicate the position of variables. $H(f)$ is 1 outside the FW-H surface $(f>0)$, and zero elsewhere $(f \leq 0)$. $p^{\prime} H(f)$ is the windowed pressure fluctuation, where $p^{\prime}$ is the fluctuating pressure. $T_{i j}, F_{i}$ and $Q$ represent the equivalent quadrupole, dipole and monopole sources respectively (more details can be found in reference 43). The Dirac delta function $\delta(f)$ implies that the thickness noise sources $(Q)$ and the loading noise sources $\left(F_{i}\right)$ are only present on the FW-H surface defined by $f=0$. The free-space Green's function is the fundamental solution of the wave equation, which satisfies the Sommerfeld radiation condition. 44 Therefore, the solution of Eq. (8) can be written as:

$$
\begin{array}{r}
p^{\prime}(\mathbf{x}, t)=\frac{\partial^{2}}{\partial x_{i} \partial x_{j}} \int_{V} \frac{T_{i j}\left(\mathbf{y}, t-|\mathbf{x}-\mathbf{y}| / c_{0}\right)}{4 \pi|\mathbf{x}-\mathbf{y}|} d \mathbf{y} \\
-\frac{\partial}{\partial x_{i}} \int_{S} \frac{F_{i}\left(\mathbf{y}, t-|\mathbf{x}-\mathbf{y}| / c_{0}\right)}{4 \pi|\mathbf{x}-\mathbf{y}|} d \mathbf{y} \\
+\frac{\partial}{\partial t} \int_{S} \frac{Q\left(\mathbf{y}, t-|\mathbf{x}-\mathbf{y}| / c_{0}\right)}{4 \pi|\mathbf{x}-\mathbf{y}|} d \mathbf{y}
\end{array}
$$

where $V$ denotes the control volume and $S$ is the FW-H surface. More details can be found in the literature [45]. In the current work, the cuboid surfaces are chosen as the FW-H surface and the quadrupole source from the turbulent flow is neglected due to the low Mach numbers studied here $\underline{41}$

\section{BENCHMARK CASES}

\section{A. Flow over a wall-mounted cube}

To gain confidence in the adopted numerical methodology for predicting the flow features accurately, a benchmark case on the flow over a wall-mounted cube at a Reynolds number of 50,000 was performed and presented in detail in reference [46] including comparisons with available measurements in the literature. The results obtained numerically agreed commendably with those from measurements both quantitatively and topologically. In particular, features of the near-wall flow patterns in this benchmark case were captured accurately, validated by the available near-wall Particle Image Velocimetry (PIV) measurements. ${ }^{47}$ In addition, to investigate the most suitable spatial resolution, 
thorough grid dependence studies were also conducted ${ }^{[6]}$ The meshing strategy adopted in the present work is based on the criteria derived from this benchmark case.

\section{B. Pressure fluctuations of a square cylinder and the far-field noise}

To the authors' best knowledge, no previous work is found about the pressure fluctuations on the surfaces of cuboids in free flow. To evaluate the feasibility of the adopted numerical method in predicting wall pressure fluctuations, a separate simulation on a square cylinder was carried out employing the same approach and reported in detail in reference [46]. A good agreement of the root-mean-square pressure $\left(p_{\text {rms }}\right)$ along the cylinder surfaces was achieved 46 between the simulation and measurements from the literature.

The far-field noise from this square cylinder was also predicted using FW-H acoustic analogy and compared with experimental data ${ }^{46}$ Discrepancies of the Overall Sound Pressure Level (OASPL) at different receivers between the predicted and measured sound were less than $1 \mathrm{~dB}$ at a flow velocity of $20 \mathrm{~m} / \mathrm{s}\left(R e=5.5 \times 10^{4}\right)$.

\section{COMPUTATIONAL SETUP}

The length, height and width of the cuboid are represented by $L, H$ and $W$ respectively. The height of the cuboid in the cross-flow direction $H$ is used as the characteristic length, and is kept constant as $75 \mathrm{~mm}$ for all cases. The aspect ratio is defined as the width of the cuboid in the spanwise direction divided by its length in the streamwise direction, namely, $W / L$. A series of aspect ratios are taken into account ranging from $1 / 6$ to $12(1 / 6,1 / 3,1 / 2,1 / \sqrt{2}, 1, \sqrt{2}, 2$, 3, 6, 12) and Fig. 1 illustrates geometries of cuboids with different aspect ratios. The origin of the coordinate system is located at the centre of cuboids, while $L, H$ and $W$ are orientated along the $x-, y-$ and $z$-axes respectively. For cases with $W / L<1$, the cuboid width $W$ in the $z$ - direction is kept the same as the height, $75 \mathrm{~mm}$, and the cuboid length $L$ in the $x$ - direction is increased accordingly as shown in Fig. 1(a). In contrast, when $W / L>1$, the length $L$ is kept constant as $75 \mathrm{~mm}$ and the width $W$ is varied to obtain different aspect ratios as illustrated in Fig. 1(c), In particular, when $W / L=1$ shown in Fig. 1(b)] the cuboid becomes a cube.

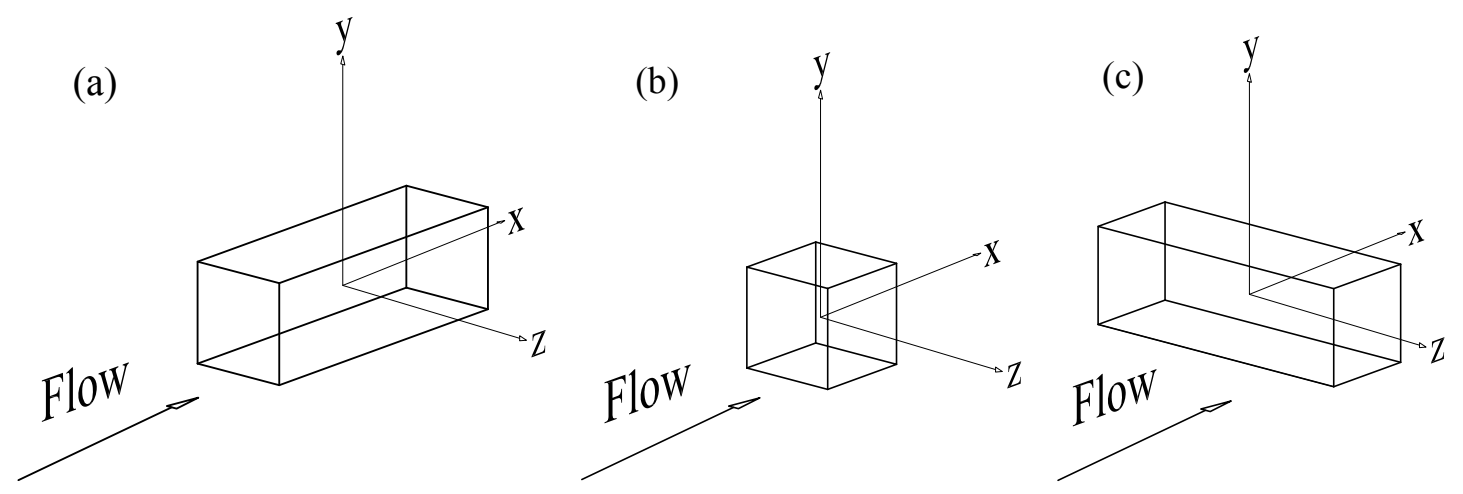

FIG. 1. Illustrations of cuboids with different aspect ratios. (a) $W / L<1$, (b) $W / L=1$ (cube), (c) $W / L>1$

A sketch of the computational domain together with the assigned boundary conditions is shown in Fig. 2 Three incoming flow velocities are considered, $U_{\infty}=10 \mathrm{~m} / \mathrm{s}, 20 \mathrm{~m} / \mathrm{s}$ and $40 \mathrm{~m} / \mathrm{s}$. The corresponding Reynolds numbers 


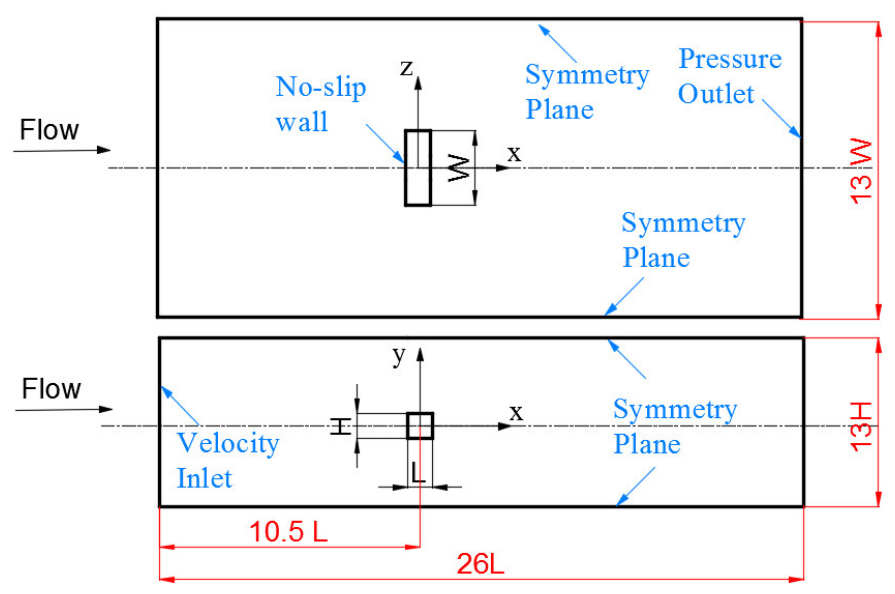

FIG. 2. Sketch and boundary conditions of the computational domain

are $R e=U_{\infty} H / \nu=50,000,100,000$ and 200,000 respectively. Since the incoming flow speed is low, the flow is assumed to be incompressible. In addition, as depicted in Fig. 2, Velocity Inlet, Pressure Outlet, Symmetry Plane and No - slipwall are assigned as the boundary conditions for the numerical simulations. The time step for all simulations is $1 \times 10^{-5} \mathrm{~s}$, with corresponding Courant-Friedrichs-Lewy (CFL) numbers smaller than 2. The overall size of the computational domain is $26 L \times 13 \mathrm{~W} \times 13 \mathrm{H}$, changing with the aspect ratio with limiting dimensions of $70 \mathrm{H}$ and $40 \mathrm{H}$ in the streamwise and spanwise directions respectively. Fully-structured meshes are generated for the computational domain. The spatial resolution is determined from the benchmark case conducted previously on a wall-mounted cube $\stackrel{46}{[6}$ Details of the grid resolution are presented in Table I] The non-dimensional first cell height at the wall $\left(y_{1}^{+}\right)$is targeted to be 1.5 , based on which the height of the first layer grid $\left(\Delta_{y 1}\right)$ normal to the wall can be calculated. The finest grids are used at the cuboid surfaces and these are progressively increased using a stretch ratio of 1.09 further from them. The minimum grid spacing along sides of the cuboid $\left(\Delta_{\min (x 1, z 1)}\right)$ is at the sharp corners, while the maximum spacing $\left(\Delta_{\max (x 1, z 1)}\right)$ is at the middle of sides. The resolution is increased gradually from the corner to the middle. For $W / L \neq 1$, the value of $L$ or $W$ is increased accordingly. Therefore, although the minimum resolution along each side is the same, the maximum value increases in proportion to the side length. Consequently,

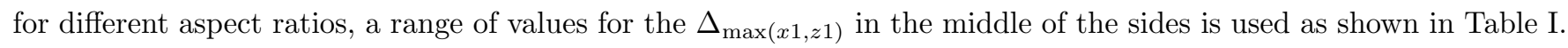

\begin{tabular}{ccccc}
\hline \hline$U_{\infty}(\mathrm{m} / \mathrm{s})$ & $\begin{array}{c}\text { targeted } \\
y_{1}^{+}\end{array}$ & $\Delta_{y 1} / H$ & $\Delta_{\min (x 1, z 1)} / H$ (corner) & $\Delta_{\max (x 1, z 1)} / H$ (middle) \\
\hline \hline 10 & 1.5 & $5.67 \times 10^{-4}$ & $2.67 \times 10^{-2}$ & $4.67-13.3 \times 10^{-2}$ \\
\hline 20 & 1.5 & $3.00 \times 10^{-4}$ & $1.84 \times 10^{-2}$ & $3.20-11.3 \times 10^{-2}$ \\
\hline 40 & 1.5 & $1.57 \times 10^{-4}$ & $0.97 \times 10^{-2}$ & $1.67-8.00 \times 10^{-2}$ \\
\hline
\end{tabular}

TABLE I. Details of the grid resolution

To gain confidence in the above described mesh resolution in addition to the case of the cube investigated in reference [46], grid dependence investigations are conducted on the bounding cases with $W / L=1 / 6$ and 12 at $R e=50,000$. The grid resolution for these two bounding cases is either coarsened and refined respectively by a ratio of $\sqrt{2}$ to obtain the new mesh. The total number of cells $\left(N_{\text {grid }}\right)$ for the coarse, original and finer meshes can be found in TableII. The 
frontal area, namely $A=W \times H$, is adopted as the reference area to calculate the drag, lift and side-force coefficients $\left(C_{d}, C_{l}, C_{s}\right.$ respectively) of the cuboids with different aspect ratios. Consequently for $W / L \leq 1, A=L^{2}$; whereas for $W / L>1, A$ increases in proportion to the aspect ratio. The mean drag coefficient $\left(\bar{C}_{d}\right)$ and root-mean-square (rms) values of these aerodynamic coefficients for the meshes with different resolutions are shown in Table II These statistics are sampled from a normalised time $t^{*}=t U_{\infty} / H=400$ to 1800 . Due to the symmetry of the geometry, the mean lift and side-force coefficients are zero. As shown in Table II] although some differences are seen between the coarse mesh and the original one, with further increase of the grid resolution, these aerodynamic coefficients only change slightly, which means the original mesh resolution in Table 1 is sufficient.

\begin{tabular}{ccccccc}
\hline \hline$W / L$ & mesh & $N_{\text {grid }}$ & $\overline{C_{d}}$ & $C_{\mathrm{d}, r m s}$ & $C_{\mathrm{l}, r m s}$ & $C_{\mathrm{s}, r m s}$ \\
\hline \hline $1 / 6$ & coarse & $1.18 \times 10^{6}$ & 1.007 & 0.0171 & 0.0523 & 0.0508 \\
\hline $1 / 6$ & original & $1.71 \times 10^{6}$ & 0.980 & 0.0138 & 0.0434 & 0.0435 \\
\hline $1 / 6$ & refined & $3.16 \times 10^{6}$ & 0.971 & 0.0116 & 0.0392 & 0.0395 \\
\hline 12 & coarse & $1.29 \times 10^{6}$ & 1.452 & 0.0897 & 0.0815 & 0.0123 \\
\hline 12 & original & $1.79 \times 10^{6}$ & 1.509 & 0.0551 & 0.0865 & 0.0119 \\
\hline 12 & refined & $3.90 \times 10^{6}$ & 1.525 & 0.0538 & 0.0857 & 0.0117 \\
\hline
\end{tabular}

TABLE II. Grid dependence investigations on the bounding cases at $R e=50,000$

For aspect ratios greater than 1, the overall grid size grows with the increase of the aspect ratio and the Reynolds number, while it also increases for smaller values of $W / L$ when the aspect ratio is smaller than unity. The overall grid sizes are similar for cases with reciprocal values of $W / L$ at the same Reynolds number. The total number of grid cells for the cuboid with $W / L=\sqrt{2}$ at $R e=50,000$ is 780,000 increasing to 7.8 million for the case with $W / L=12$ at $R e=200,000$. The maximum value of $y_{1}^{+}$is less than 2 , while most of the values are smaller than 1 with an average of about 0.7 . Distributions of $y_{1}^{+}$around the cuboid with $W / L=12$ at $R e=200,000$ are shown in Fig. 3 as examples.
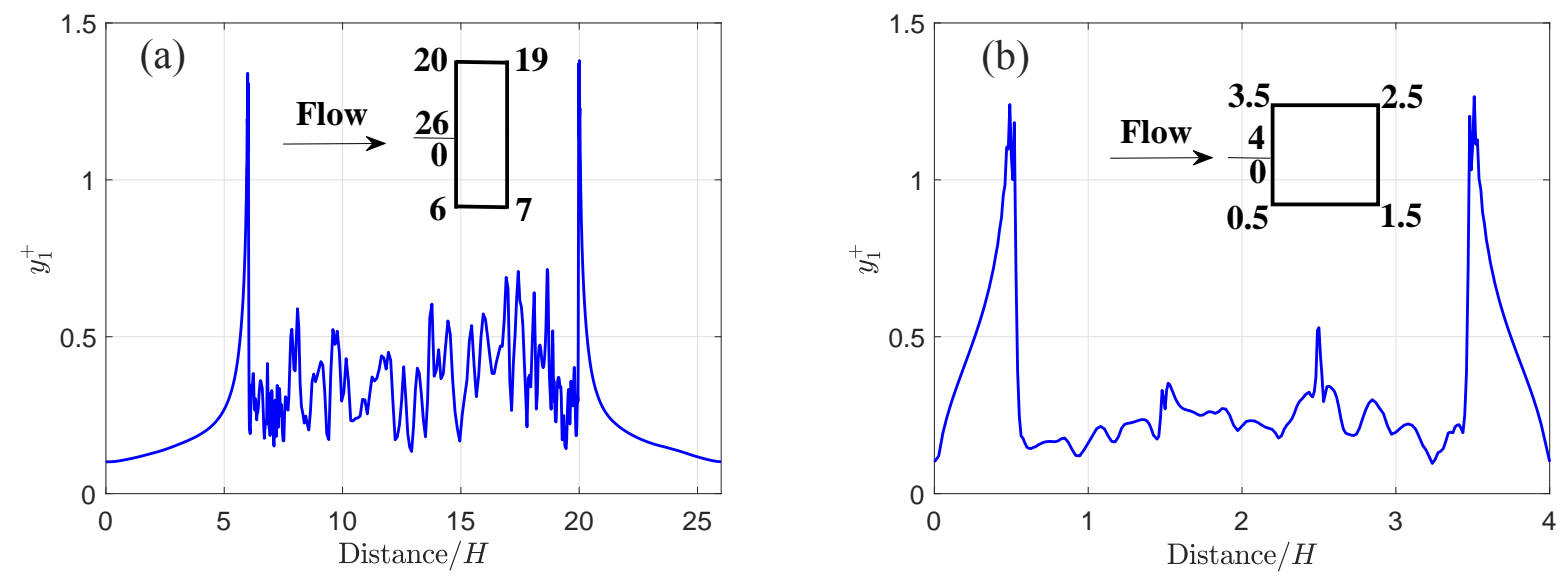

FIG. 3. The distribution of $y_{1}^{+}$along the cuboid with $W / L=12$ at $R e=200,000$. (a) $y=0$ plane, (b) $z=0$ plane 


\section{EFFECT OF THE ASPECT RATIO ON THE FLOW}

\section{A. Aerodynamic coefficients}

Fig. 4 shows examples of the time histories of $C_{d}, C_{l}$ and $C_{s}$ at $R e=100,000$ from $t^{*}=820$ to 900 for representative cuboids with $W / L=1 / 6,1 / \sqrt{2}, 1, \sqrt{2}, 6$, and 12 . The main features of the corresponding time histories at $R e=$ 50,000 and 200,000 are similar to those observed in Fig. 4. As can be seen, the mean lift and side-force coefficients are very close to zero due to symmetry of the objects, while the mean drag coefficient is close to 1 . In addition, once the aspect ratio exceeds 1 , the drag coefficient grows slightly for larger $W / L$, even after allowing for the larger reference area $A$. With the increase of the aspect ratio, fluctuations of the lift coefficient change more significantly than those of the drag and side-force coefficients. Higher frequency fluctuations of $C_{l}$ are seen for cuboids with $W / L<1$, while the highest amplitude of $C_{l}$ is observed in the case with $W / L=6$.
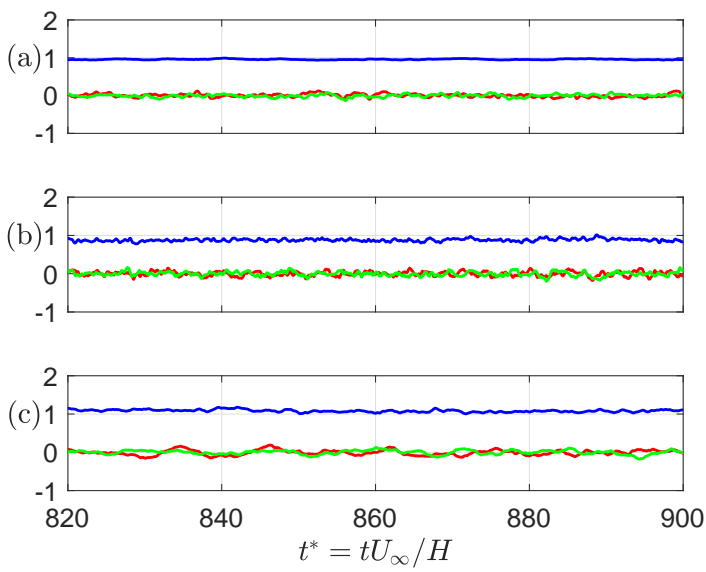
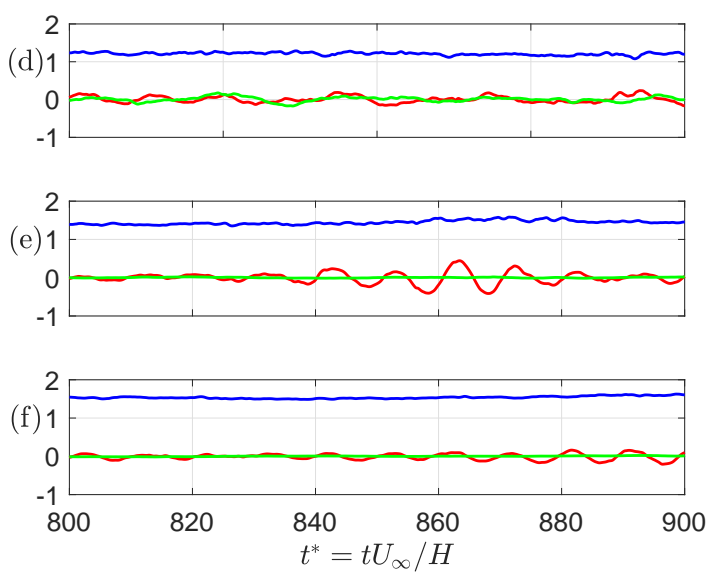

FIG. 4. Time histories of $C_{d}$ (blue), $C_{l}$ (red) and $C_{s}$ (green) at $R e=100,000$ for aspect ratios plotted against normalized time $t^{\star}$. From (a) to (f), $W / L=1 / 6,1 / \sqrt{2}, 1, \sqrt{2}, 6$, and 12 , respectively

The mean value of drag coefficient and rms values of these aerodynamic coefficients for cuboids with all the studied aspect ratios are presented in Fig. 5. These are calculated from time histories sampled from $t^{*}=400$ to 2400 at different Reynolds numbers. It has been checked that the statistics have converged within this time. The trends of these aerodynamic coefficients with respect to the aspect ratio are similar at different Reynolds numbers. The mean drag coefficient is more independent of Reynolds numbers than the rms values of $C_{d}, C_{l}$ and $C_{s}$, while the rms values tend to drop slightly at a higher Reynolds number.

For cuboids with $W / L<1$, the mean drag coefficient of the cuboid reduces with the increase of $W / L$ and reaches the lowest value for $W / L=1 / \sqrt{2}$, as shown in Fig. 5(a). Further analysis of the flow field (given in next section) shows that the separated flow from the leading edge in this case reattaches at the trailing edge. The value of $\bar{C}_{d}$ increases considerably from $W / L=1 / \sqrt{2}$ to 1 and it grows for further increase of $W / L$. The rms values of the drag and lift coefficients shown in Figs $5(\mathrm{~b})$ and $5(\mathrm{c})$ increase from $W / L=1 / 6$ to 6 but drop at $W / L=12$. According to Fox and West, $\frac{48}{1}$ the 'infinite' condition of the cylinder can be achieved for aspect ratios greater than 30 . Therefore, the results obtained from the current work cannot be compared directly with those of the infinite cylinder! 1 The results for $C_{s, \mathrm{rms}}$ in Fig. $5(\mathrm{~d})$ show that, with the increase of $W / L$, it grows first to a maximum for the cube with 

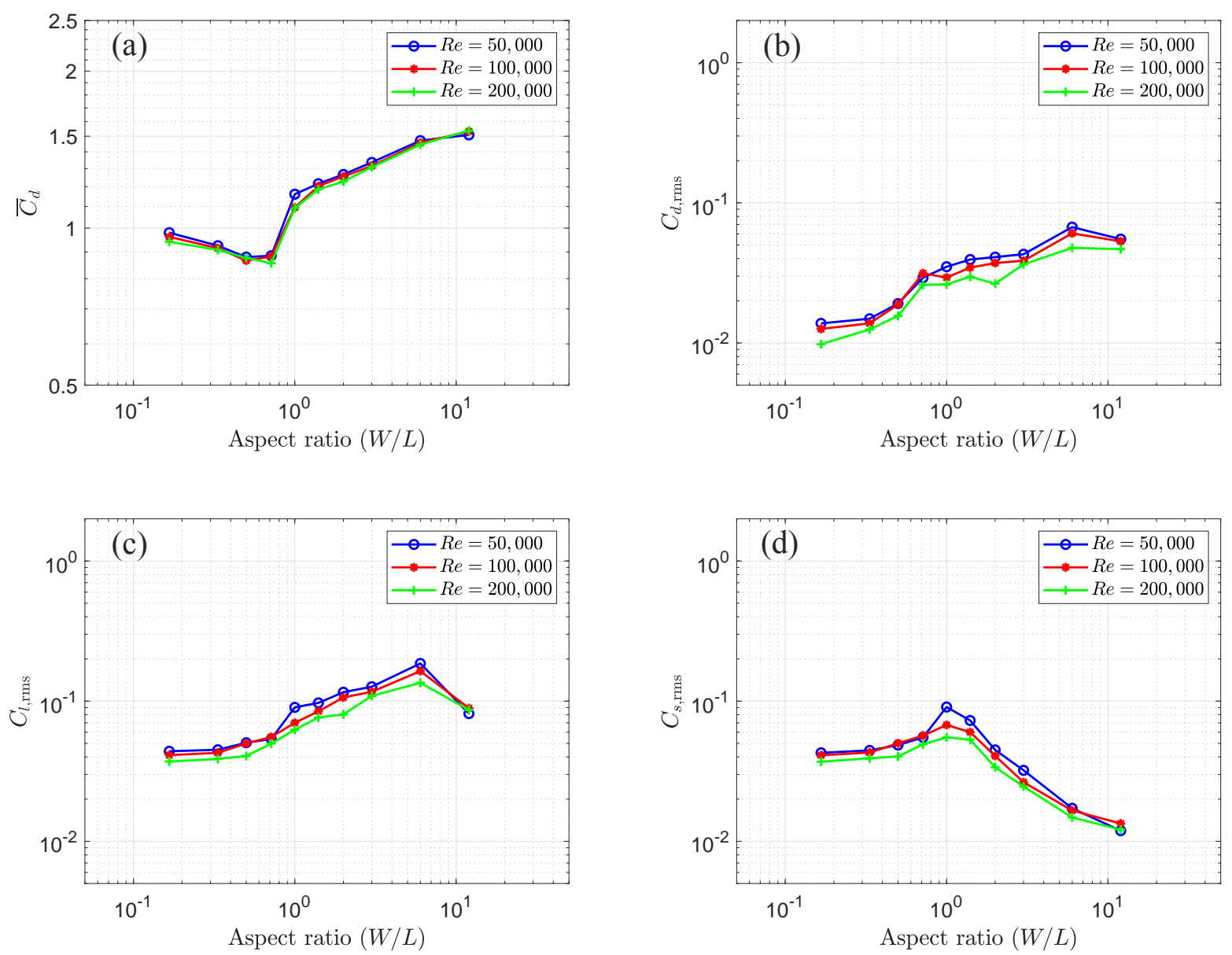

FIG. 5. The trend of aerodynamic coefficients changing with the aspect ratio at three Reynolds numbers. (a) $\bar{C}_{d}$, (b) $C_{d, \mathrm{rms}}$, (c) $C_{l, \mathrm{rms}}$, (d) $C_{s, \mathrm{rms}}$

$W / L=1$. For $W / L>1, C_{s, \text { rms }}$ reduces roughly in proportion to $W / L$, since rms values of the side force remain roughly constant while the normalising area increases.

The Power Spectral Densities (PSDs) of these aerodynamic coefficients are presented in Fig. 6 for cuboids with representative aspect ratios at $R e=100,000$ as examples. The spectra at the other Reynolds numbers show similar trends. These are obtained using Welch's method. A Hanning window is applied with $50 \%$ overlapping and the frequency resolution is about $1.22 \mathrm{~Hz}(S t \approx 0.005)$. The shapes of the PSDs for cuboids with $W / L<1$ are similar to each other so only the bounding cases with $W / L=1 / 6$ and $1 / \sqrt{2}$ are displayed as examples in Fig. 6. Force spectra of the cuboids with $W / L=\sqrt{2}, 6$ and 12 are also shown, while those for $W / L=2$ and 3 lie between them and have similar features.

No evident peaks can be observed in spectra of $C_{d}, C_{l}$ and $C_{s}$ for $W / L<1$. The PSDs of $C_{d}$ for cuboids with $W / L<1$ increase gradually at Strouhal numbers greater than 0.08 as the aspect ratio is increased, while the spectra of the lift and side-force coefficients are almost unchanged for cases with $W / L<1$. Although the PSDs of the drag coefficient are also broadband for cases with $W / L \geq 1$ in Fig. 6(a). distinct peaks centred close to $S t=0.1$ can be seen in the spectra of $C_{l}$ in Fig. 6(b) while the peak Strouhal number of a square cylinder is close to $0.13^{22}$ These become narrower as the values of $W / L$ are increased. In Fig. 6(c), broadband peaks occur in the PSDs of $C_{s}$ for 

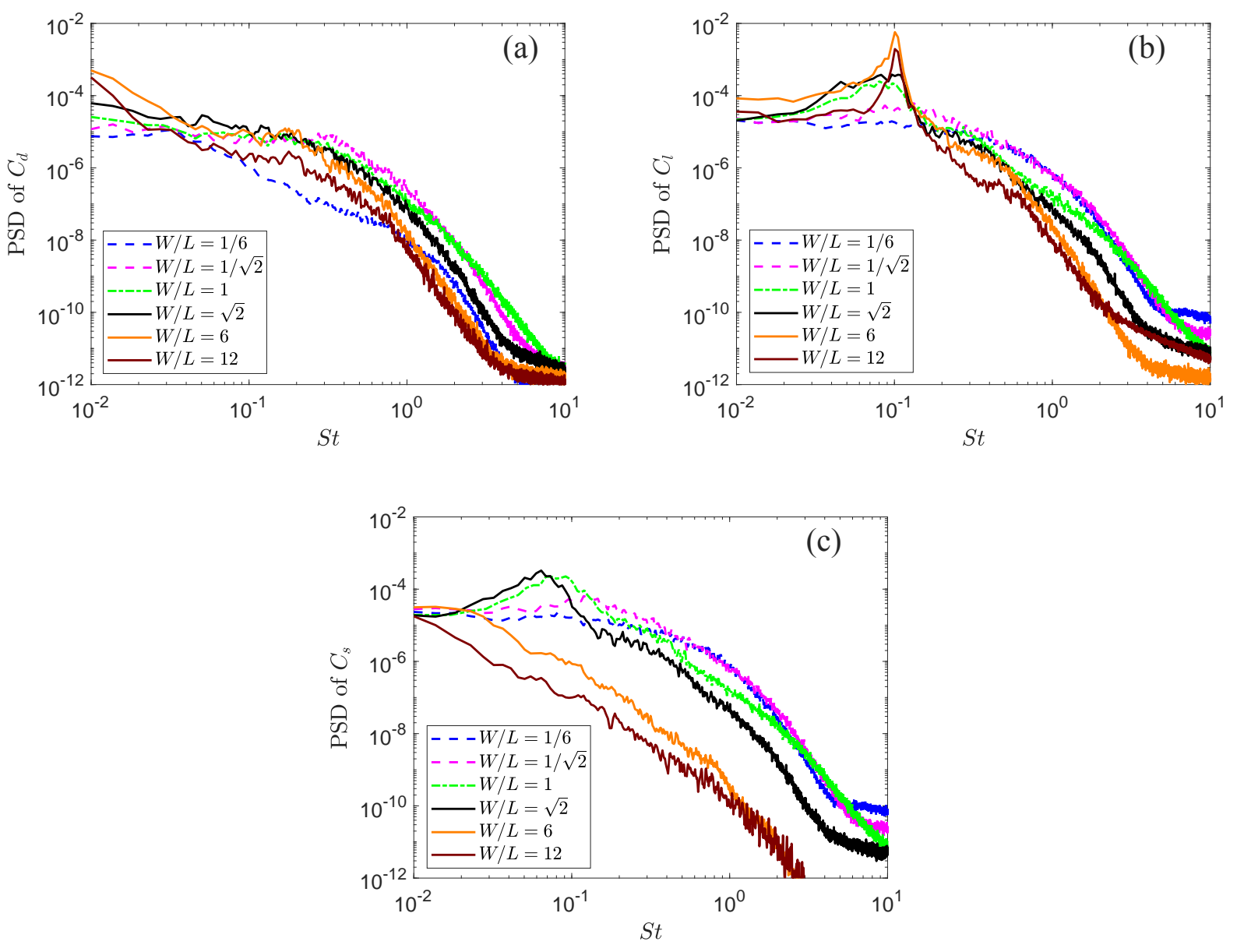

FIG. 6. PSDs of (a) $C_{d}$, (b) $C_{l}$, (c) $C_{s}$ at $R e=100,000$ for different aspect ratios

cuboids with $W / L=1$ and $\sqrt{2}$. However, with the further increase of the aspect ratio, the broadband peaks disappear and the amplitudes of the spectra drop rapidly due to the effect of the normalising area. Further explanations about above described features of the PSDs can be found in Section V.B.1, related with the flow patterns.

\section{B. Flow patterns around the cuboid}

\section{Instantaneous flow structures}

To illustrate the flow behaviour around these cuboids, Fig. 7 7 shows the normalised instantaneous spanwise vorticity $\left(\omega_{z}\right)$ for representative aspect ratios. The flow patterns around these cuboids are almost the same at the different Reynolds numbers, so only those at $R e=100,000$ are presented in the following sections as examples. It can be seen that the flow always separates from the leading edges of the cuboids, which also happens at $R e=50,000$ and 200, 000. However, as can be seen in Fig. 7, the flow structures around these cuboids change significantly with the aspect ratio. For $W / L<1$, the separated flow breaks into intensive and irregular vortices around the cuboid; these vortices are convected downstream and dissipated rapidly. Few of them reach the rear of the cuboid and no periodic vortex shedding is formed in the wake. Therefore, no peaks can be seen in the spectra in Fig. 6 for $W / L<1$. With 
the reduced value of $L$ (still with $W / L<1$ ), the intensity of vortices formed downstream the cuboid increases, leading to higher amplitudes of $C_{d}$ spectra in Fig. 6(a). In contrast, when the aspect ratio exceeds unity, the separated flow becomes more and more coherent with the increase of $W / L$, due to the weakened flow interactions from the lateral sides of the cuboid. The separated flow convects to the wake of the cuboid and starts to shed alternately along the cross-flow direction, which explains the distinct peaks in $C_{l}$ observed in Fig. 6(b) for cuboids with $W / L>1$.
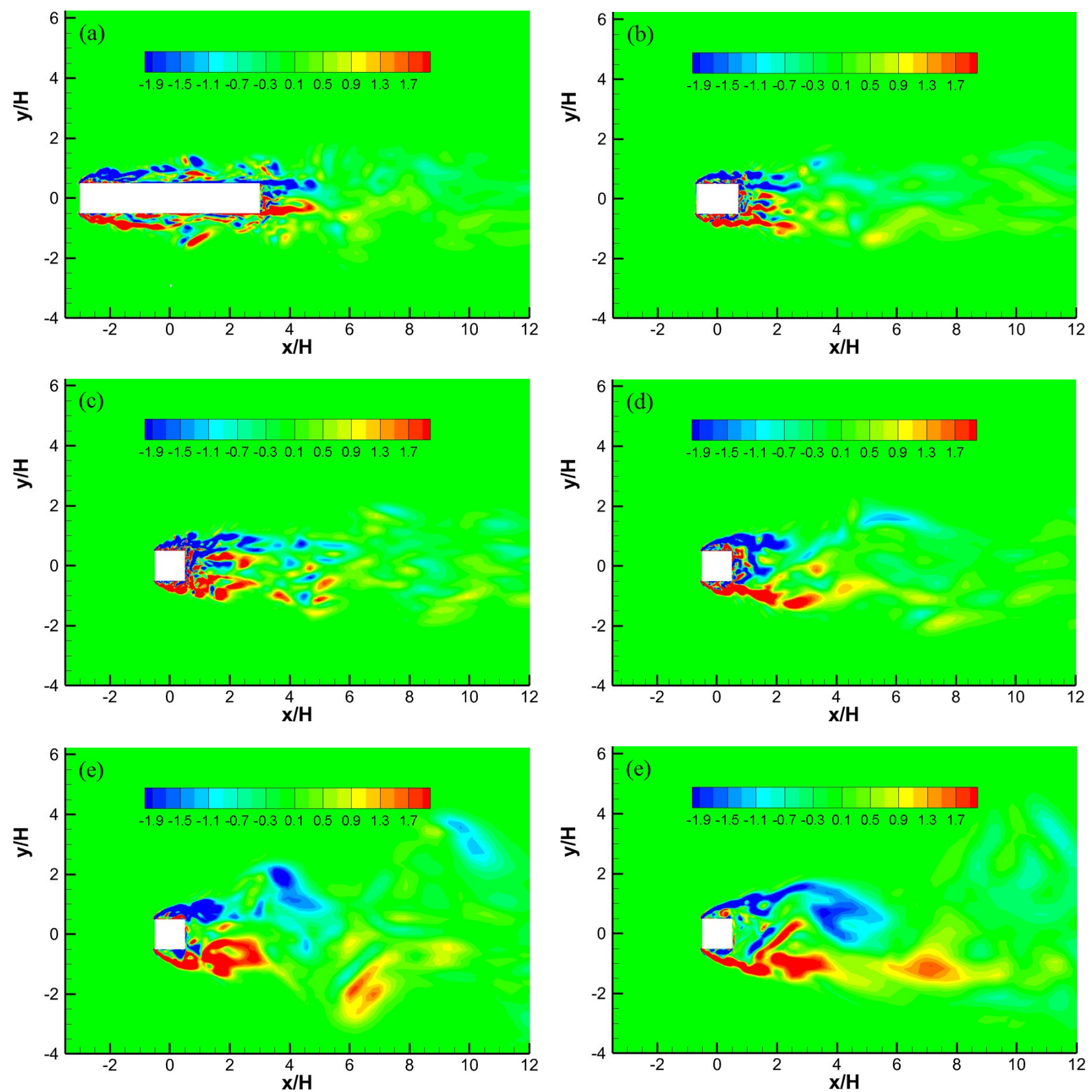

FIG. 7. Contours of the instantaneous spanwise vorticity for cuboids with different aspect ratios at a Reynolds number of 100,000. (a) $W / L=1 / 6$, (b) $W / L=1 / \sqrt{2}$, (c) $W / L=1$, (d) $W / L=\sqrt{2}$, (e) $W / L=6$, (f) $W / L=12$ 


\section{Time-averaged flow patterns in the $z=0$ plane}

To characterise the time-averaged flow patterns surrounding these cuboids, mean streamlines in the $z=0$ plane are shown in Fig. 8 for representative aspect ratios, superposed by contours of the time-averaged pressure coefficient. Fig. 9 presents close-up views of the flow patterns near the top surface of the cuboid. Since symmetric mean flow patterns are observed, indicating convergence of the numerical calculations, only the parts above $y / H=0$ are displayed in these figures. The thick white lines in Figs 8 and 9 denote locations of zero streamwise velocity, which indicates flow separation, reattachment and the recirculation length in the wake. As can be seen from Fig. 8 , the incoming flow always separates at the leading edge of the cuboid. However, the recirculation length in the wake of these cuboids changes significantly with the variation of $W / L$, implied by the position of the white thick lines intersecting with $y / H=0$.
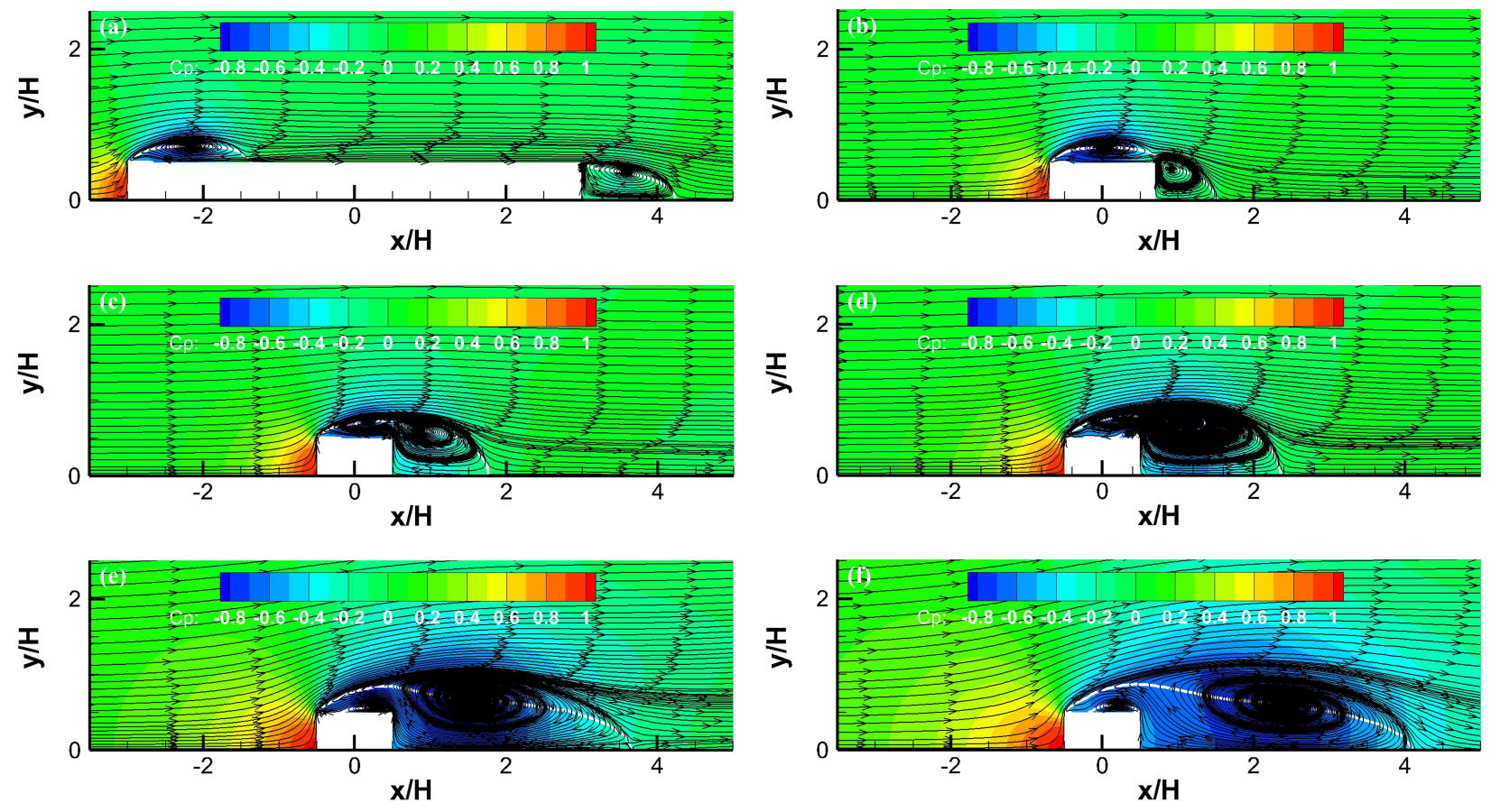

FIG. 8. Time-averaged pressure distribution contour together with mean streamlines in the $z=0$ plane for cuboids with different aspect ratios at a Reynolds number of 100,000. (a) $W / L=1 / 6$, (b) $W / L=1 / \sqrt{2},(\mathrm{c}) W / L=1,(\mathrm{~d}) W / L=\sqrt{2},(\mathrm{e})$ $W / L=6$, (f) $W / L=12$

As shown in Figs 8(a) and 8(b) for $W / L<1$ the flow separated from leading edges reattaches to the top surface and remains attached until reaching the trailing edges. The position of the flow reattachment moves towards the trailing edge of the cuboid as the aspect ratio is increased from $W / L=1 / 6$ to $1 / \sqrt{2}$. This can be seen from the close-up view of the flow pattern near the cuboid with $W / L=1 / \sqrt{2}$ in Fig. $9(\mathrm{a})$. However, for $W / L \geq 1$, shown in Figs 8(c)-8(f), instead of reattaching to the top surface, the separated flow is convected downstream to the wake and forms a large recirculation region behind the cuboid. As highlighted by the red dotted rectangles in Figs 9(b)- $9(\mathrm{e})$, secondary vortices can be seen close to the trailing edges, the size of which changes considerably for different aspect ratios. Quantitative descriptions of the recirculation length in the wake, the flow reattachment on the top surface for $W / L<1$ and also the secondary vortices for $W / L \geq 1$ will be presented in the following Section V.B.3. Implications 

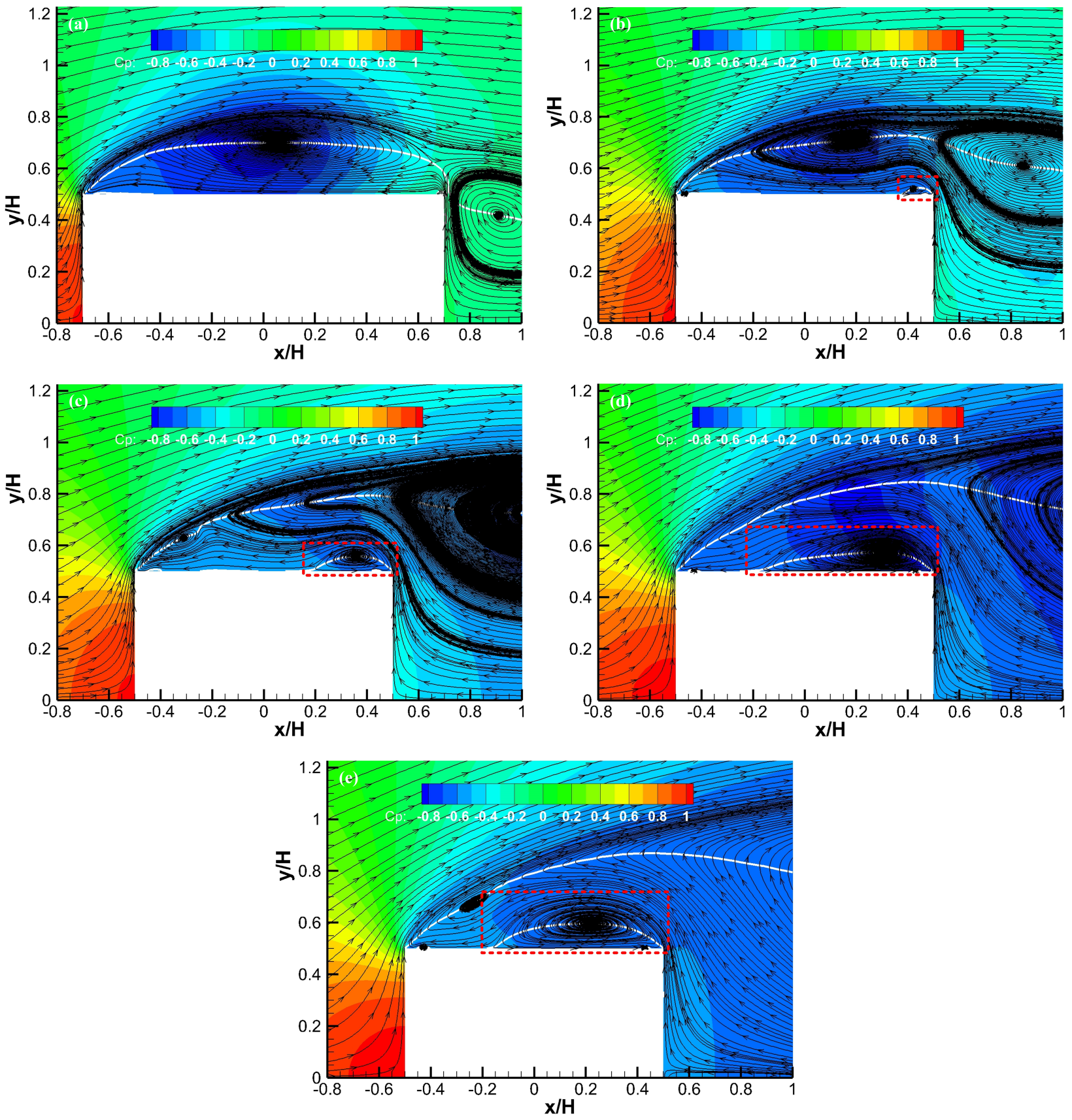

FIG. 9. A close-up view of the flow pattern in the $z=0$ plane near the cuboids, coloured by the pressure coefficient at a Reynolds number of 100,000 . (a) $W / L=1 / \sqrt{2}$, (b) $W / L=1$, (c) $W / L=\sqrt{2}$, (d) $W / L=6$, (e) $W / L=12$

of the flow patterns for the noise radiation is discussed in terms of the surface pressure fluctuations in Section V.D.

In summary, the flow always separates from the cuboid leading edge. For aspect ratios smaller than 1, the separated flow reattaches to the lateral surfaces and the location of the flow reattachment moves to the trailing edge at $W / L=$ $1 / \sqrt{2}$. However, no flow reattachment can be observed on the top and bottom surfaces for $W / L \geq 1$; the separated flow is convected downstream to the wake with a secondary vortex formed near the trailing edge. The size of the secondary vortex for aspect ratios greater than 1, and the recirculation length in the wake of all the presented cases in Fig. 8 are significantly affected by the variation of the aspect ratio, more details of which will be discussed 
quantitatively in the following part of this section.

\section{Recirculation length in the wake and the separation bubble on the top surface}

The recirculation length $L_{r}$ is measured from the rear surface of the cuboid to the location where the negative streamwise velocity in the wake recirculation flow recovers to zero. Therefore, the velocity profiles along the centreline ( $y=0, z=0$ ) in the wake of the cuboid can be used to determine the values of $L_{r}$. Fig. 10(a) shows these mean streamwise velocity profiles for representative cuboids at $R e=100,000$ as examples, which are almost the same as those at $R e=50,000$ and 200,000. The recirculation length is implied by the distance between the start point of these profiles and the intersection point with $U / U_{\infty}=0$.

A summary of the recirculation lengths of all the studied cuboids is presented in Fig. 10(b). The trend of $L_{r}$ with respect to the aspect ratios is independent of the Reynolds number. The lowest value of $L_{r}$ is found in the case with $W / L=1 / \sqrt{2}$. The recirculation length drops first from $W / L=1 / 6$ to $1 / \sqrt{2}$, then increases quickly with the further increase of $W / L$. Again, due to the limited aspect ratios studied in this work, values of $L_{r}$ in Fig. 10(b) cannot be compared directly with that of the infinite square cylinder.2]
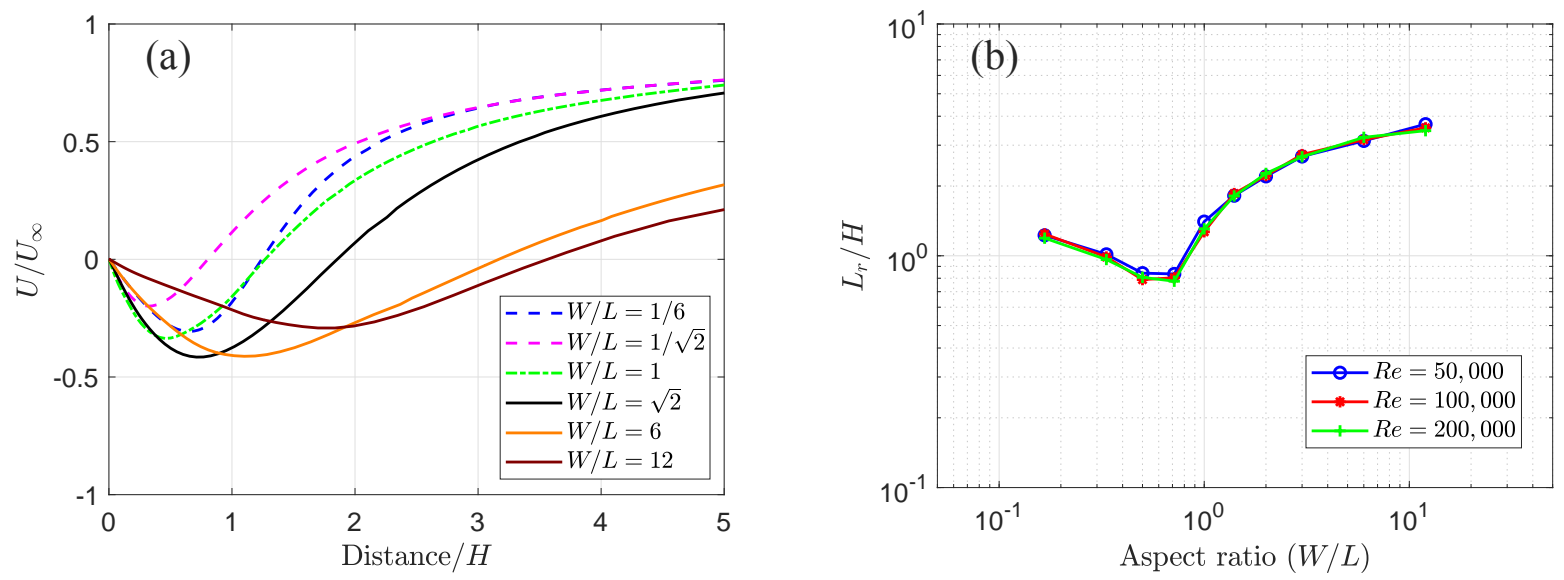

FIG. 10. (a) Velocity profiles along the centreline $(y=0, z=0)$ and (b) the recirculation length in the wake of the cuboids

Fig. 11 summarises the length of the separation bubble on the lateral surface in the $z=0$ plane. For $W / L<1$, in Fig. 11(a), this is the distance between the leading edge and the location where the separated flow reattaches to the top surface $\left(L_{r e a}\right)$. For $W / L \geq 1$, in Fig. 11(b)] it is the length of the secondary vortex, measured from the trailing edge to its end on the top surface $\left(L_{s e c}\right)$. The position at which the skin friction coefficient becomes zero $\left(C_{f}=0\right)$ along the cuboid is used to determine these values, combined with inspection of the white lines shown in Figs 8 and 9.

As shown in Fig. 11, the trend of the reattachment length with respect to the aspect ratio is consistent at different Reynolds numbers. However, the values are clearly dependent on $R e$. For $W / L<1$ in Fig. 11(a), $L_{r e a}$ reduces with the increase of both the aspect ratio and the Reynolds number, although $L_{r e a}$ corresponds to the trailing edge for $W / L=1 / \sqrt{2}$, at which it is independent of Re. In Fig. 11(b) for $W / L \geq 1, L_{\text {sec }}$ grows rapidly from $W / L=1$ to 2, while values for larger aspect ratios are greater than $0.7 H$ and only change slightly. It is noteworthy that although 

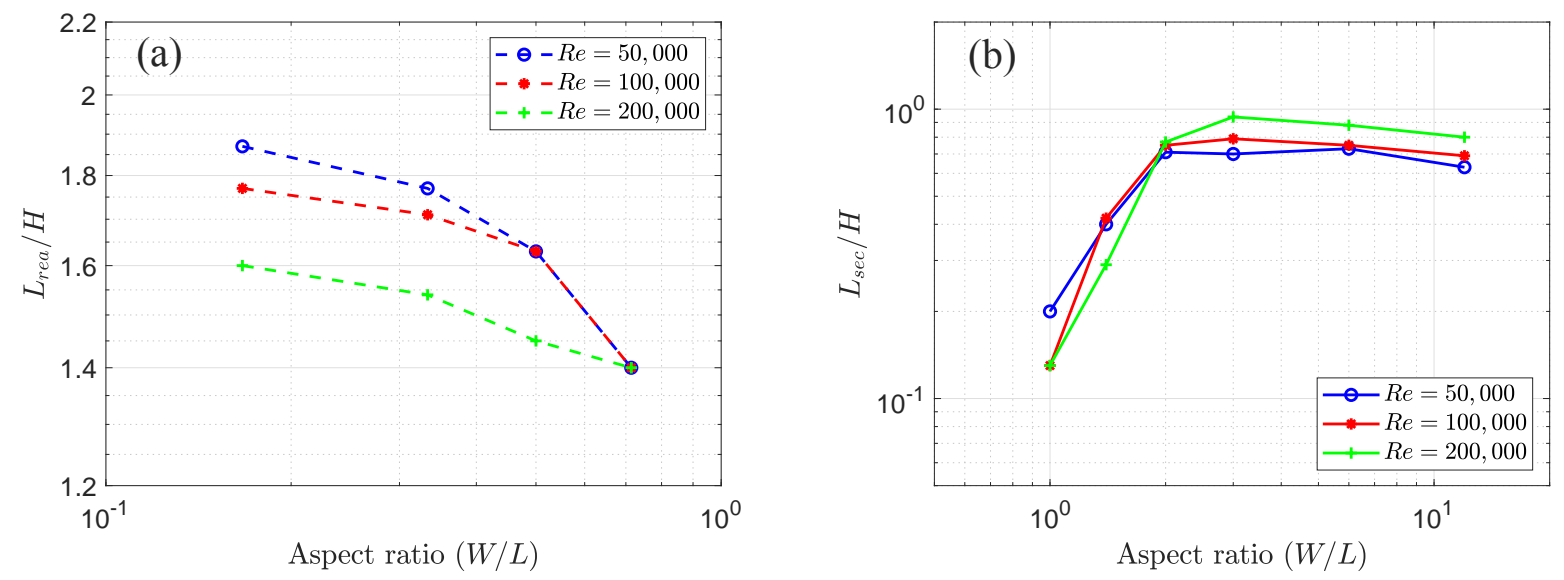

FIG. 11. Lengths of the flow reattachment in the $z=0$ plane on the lateral surface of cuboids with different aspect ratios. (a) $W / L<1$, (b) $W / L \geq 1$

the length $L_{\text {sec }}$ does not change much for $W / L \geq 2$, the height of the secondary vortex expands gradually with the increase of the aspect ratio, as illustrated in Figs $9(\mathrm{~d})$ and $9(\mathrm{e})$

\section{Time-averaged flow patterns in the $y=0$ plane}

Flow patterns in the $y=0$ plane are the same as those in the $z=0$ plane for the cuboids with $W / L \leq 1$ due to the symmetry of the geometries. However, once the aspect ratio exceeds 1 , the flow structures in the $y=0$ plane start to differ from those in the $z=0$ plane discussed above. Fig. 12 presents mean streamlines around cuboids with $W / L=\sqrt{2}, 6$ and 12 in the $y=0$ plane together with contours of the mean pressure coefficient, while Fig. 13 shows close-up views of the flow patterns near the lateral surfaces. White lines in Figs 12 and 13 indicate locations with zero streamwise velocity, as supplementary visualization of the flow.

As shown in Fig. 12 the separated flow moves downstream to the wake and forms a recirculation region labelled as ' $\mathrm{T}$ '. With the increase of the aspect ratio from $W / L=\sqrt{2}$ to 12 , the size of the vortex ' $\mathrm{T}$ ' first increases and then decreases, with its position moving towards the trailing edge. Similar to the secondary vortex observed in the $z=0$ plane in Figs 9(b)-9(e), a secondary vortex formed due to the reverse flow from the wake can also be seen in the $y=0$ plane close to the trailing edges for $W / L$ from $\sqrt{2}$ to 3 . An example for $W / L=\sqrt{2}$ is shown in Fig. 13(a) and the secondary vortex is highlighted by the red dotted rectangle. However, the secondary vortex in the $y=0$ plane moves off the lateral surface as the aspect ratio is increased from 3 to 6 and a small vortex labelled as ' $\mathrm{A}$ ' is formed in Fig. 13(b) For further increased aspect ratio of 12, the secondary vortex disappears, as shown in Fig. 13(c), which implies the reverse flow from the wake becomes too weak to reach the lateral surface due to the enlarged distance along the spanwise direction. 

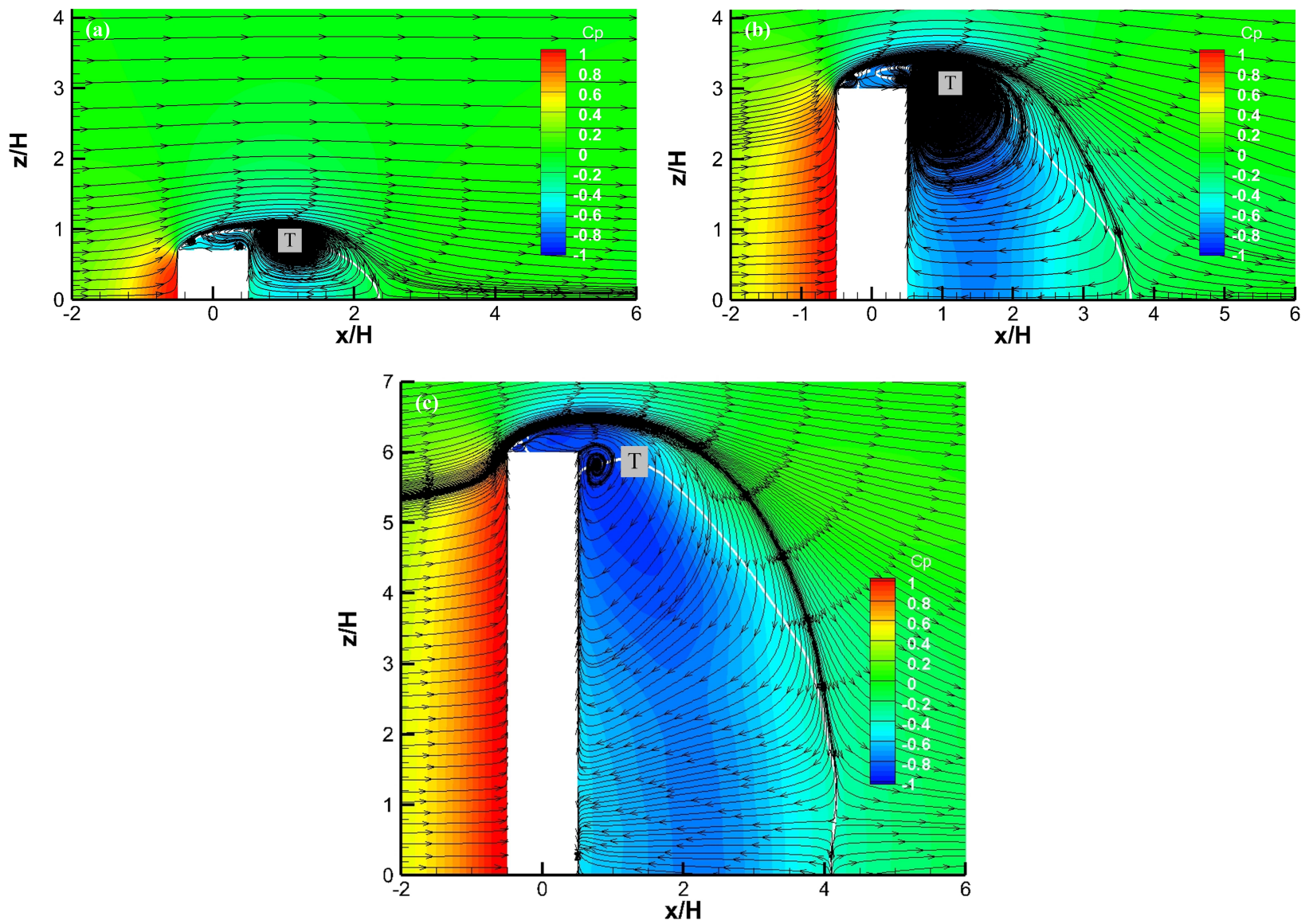

FIG. 12. Time-averaged pressure distribution contour together with mean streamlines in the $y=0$ plane for cuboids with $W / L>1$ at a Reynolds number of 100,000 . (a) $W / L=\sqrt{2}$, (b) $W / L=6$, (c) $W / L=12$

\section{Mean pressure coefficients along the cuboid surfaces}

The distribution of the surface pressure is closely related to the flow patterns described above. Fig. 14 presents the pressure coefficient around surfaces of the cuboids at $R e=100,000$. Similar results are found at $R e=50,000$ and 200,000 (not shown here). The pressure distributions in Fig. 14 start from the stagnation point on the frontal surface. The distance along each surface is normalised by the corresponding length of the cross-section as illustrated in Fig. 14(a) Fig. 14(b) displays the pressure distribution in the $z=0$ plane for $W / L \leq 1$, which is the same as that in the $y=0$ plane. As shown in Fig. 14(b), pressure distributions on the frontal surface from 0 to 0.5 for $W / L \leq 1$ are almost the same in each case while the pressure distributions on the rear surface from 1.5 to 2 are similar for each case with $W / L<1$ but are higher than for the cube. A pressure plateau is found on the top surface for $W / L=1 / 6$, indicated by the blue dashed line. The pressure increases quickly from a negative value and is almost level for the remaining part of the top surface. The negative pressure on the top surface is caused by the separated flow from the leading edge and the pressure recovers quickly because of the flow reattachment, downstream of which the pressure changes only slightly. With the increase of the aspect ratio from $1 / 6$ to $1 / \sqrt{2}$, the size of the level pressure region on the top surface diminishes relative to the length $L$; particularly for $W / L=1 / \sqrt{2}$, it disappears because the separated flow reattaches to the trailing edge as shown in Fig. $9(\mathrm{a})$. For $W / L=1$, the separated flow no longer reattaches to 

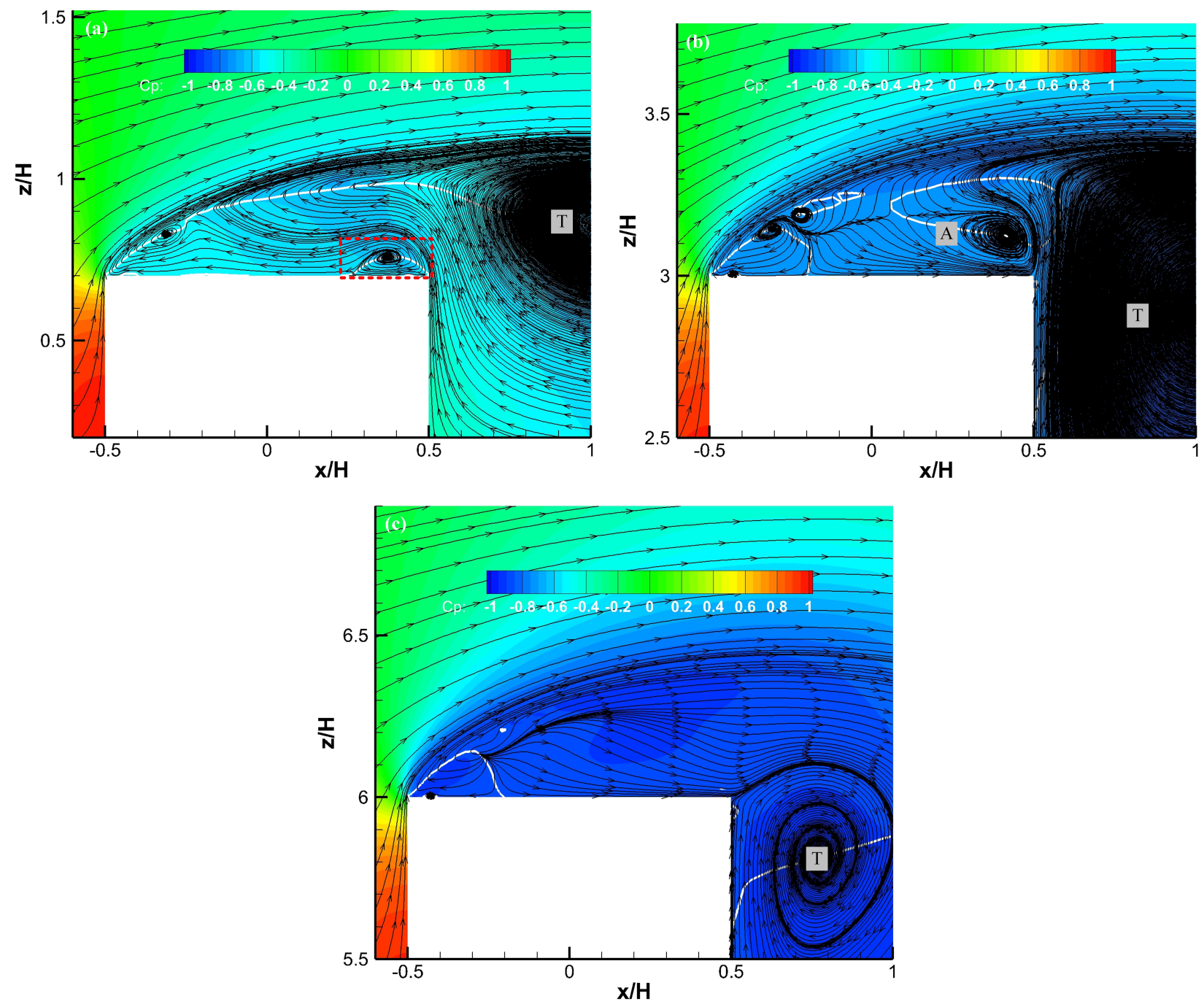

FIG. 13. Close-up views of the flow pattern in the $y=0$ plane near the cuboids, coloured by the pressure coefficient at a Reynolds number of 100,000 . (a) $W / L=\sqrt{2}$, (b) $W / L=6$, (c) $W / L=12$

the top surface and the pressure hardly changes on the top surface as denoted by the dash-dot line.

Fig. 14(c) illustrates pressure distributions in the $z=0$ plane for cuboids with representative aspect ratios $(W / L \geq$ 1). Again, the pressure on the frontal surface is almost the same in each case, whereas, the pressure on the top and rear surfaces is more sensitive to the aspect ratio. The pressure distribution on the top surface for $W / L \geq 1$ is much flatter than that for $W / L<1$ due to the disappearance of the flow reattachment and it changes only slightly for different aspect ratios. In addition, for $W / L \geq 1$, the pressure on the rear surface tends to drop gradually with the increase of the aspect ratio. Distributions of $C_{p}$ in the $y=0$ plane for representative aspect ratios are shown in Fig. 14(d). For $W / L>1$, the pressure on the lateral surface drops considerably as the aspect ratio is increased due to the development of the edge vortex shown in Fig. 13 . In addition, it is interesting to note that for cases with a secondary vortex formed on the cuboid surface close to the trailing edge $(W / L \geq 1$ in the $z=0$ plane and $1 \leq W / L \leq 3$ in the $y=0$ plane), a steep increase of the pressure can be seen at the position 1.5. 
(a)

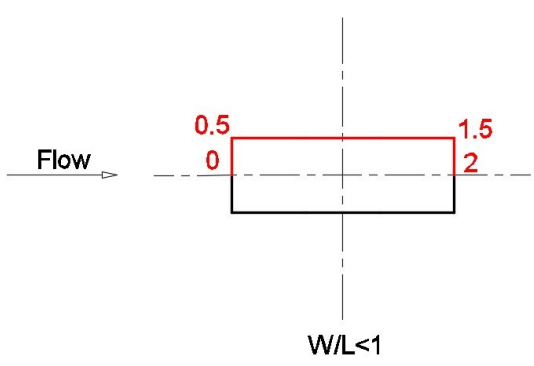

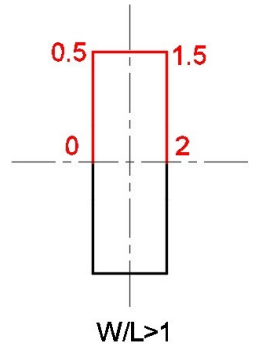

W/L>1
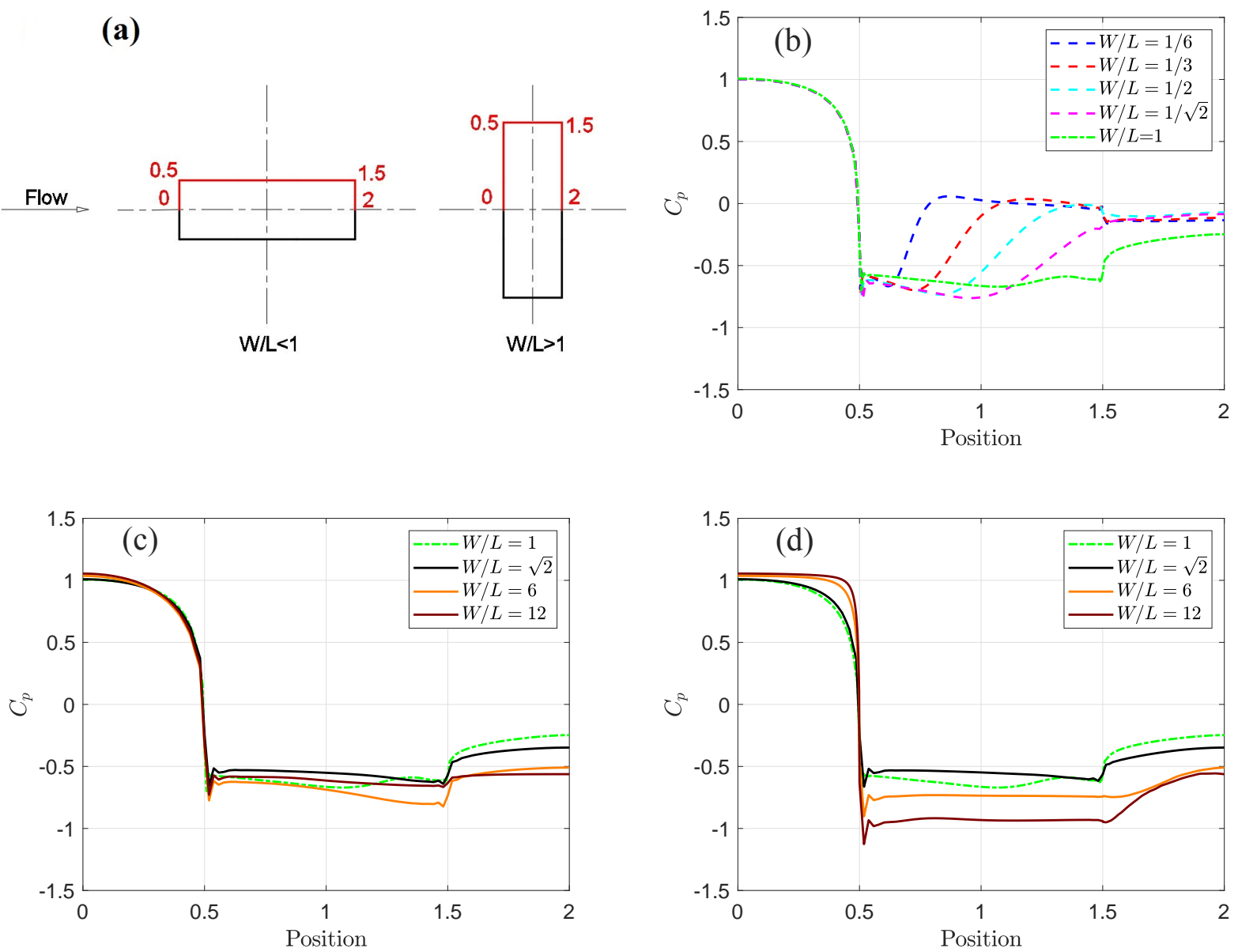

FIG. 14. Distributions of the mean pressure coefficient for the cuboids at $R e=100,000$. (a) Illustration of the normalised position, (b) $W / L \leq 1$ in the $z=0$ plane, (c) $W / L \geq 1$ in the $z=0$ plane, (d) $W / L \geq 1$ in the $y=0$ plane 
A summary of the base pressure coefficient $\left(C_{p b}\right)$ for all cases is presented in Fig. 15 , while the measured $C_{p b}$ of -1.35 for a square cylinder at $R e=100,000$ can be found in the literature [1. It turns out that the effect of Reynolds number on $C_{p b}$ is negligible. The values of $C_{p b}$ increase gradually as the aspect ratio is increased from $1 / 6$ to $1 / \sqrt{2}$, but drop significantly from $1 / \sqrt{2}$ to 1 . For larger values of $W / L$, the base pressure coefficient keeps dropping. In addition, the trend of $C_{p b}$ with respect to the aspect ratio is the opposite to that of the mean drag coefficient (Fig. $5(\mathrm{a})$.

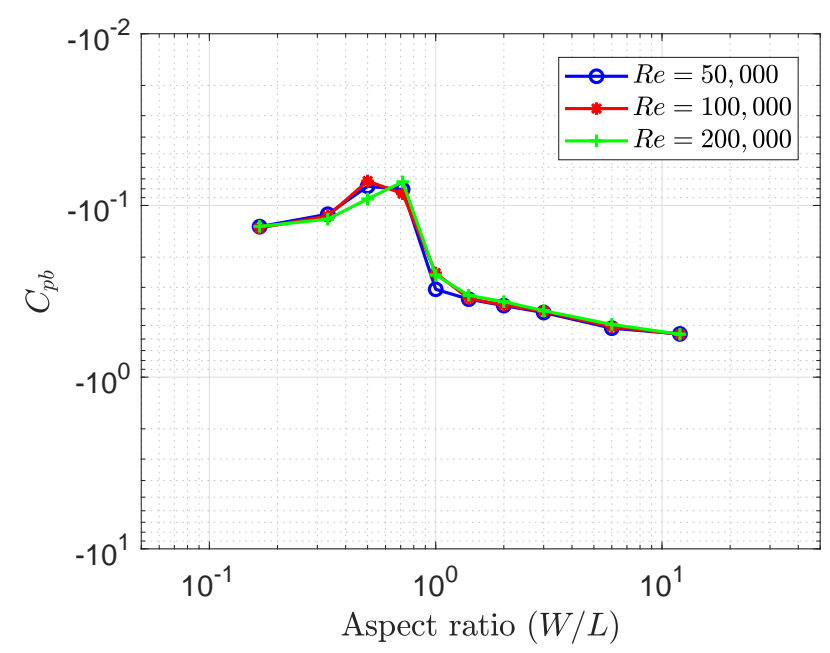

FIG. 15. The base pressure coefficient for cuboids with different aspect ratios.

\section{Pressure fluctuations on the cuboid surfaces}

Fig. 16 displays contours of the pressure fluctuations on the cuboid surfaces at $R e=100,000$, where the rms values of the pressure fluctuation are expressed in decibels:

$$
L_{p}=10 \log _{10}\left(\frac{p_{\text {rms }}}{p_{\text {ref }}}\right)^{2}
$$

where $p_{\text {ref }}=2 \times 10^{-5} \mathrm{~Pa}$ is the reference sound pressure. With the increase of the aspect ratio, the pressure fluctuations on the rear surface increase accordingly. For $W / L<1$, large pressure fluctuations occur within the flow recirculation region on the top and the lateral surfaces as illustrated in Figs 16(a) and 16(b) For $W / L=1$ in Fig. 16(c) regions with large pressure fluctuations on the top and lateral surfaces decrease with the disappearance of the flow reattachment and the highest values of $p_{\text {rms }}$ are observed close to the trailing edges. The distribution of the pressure fluctuations on the top and lateral surfaces for $W / L$ between $\sqrt{2}$ and 3 is similar to that of the cube. However, the overall level of the pressure fluctuations on the cuboid surfaces for $W / L=6$ and 12 is much higher than that for smaller aspect ratios, as can be observed in Fig. 16(e) and 16(f), while the highest value of $p_{\text {rms }}$ is again found near the trailing edge. 
(a)

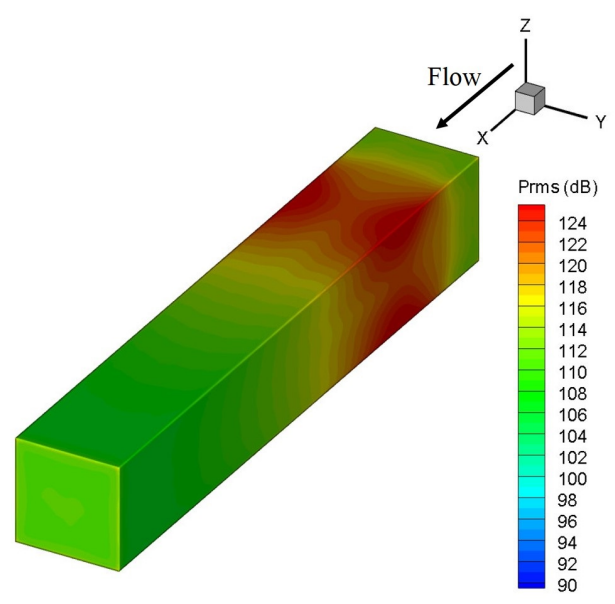

(c)

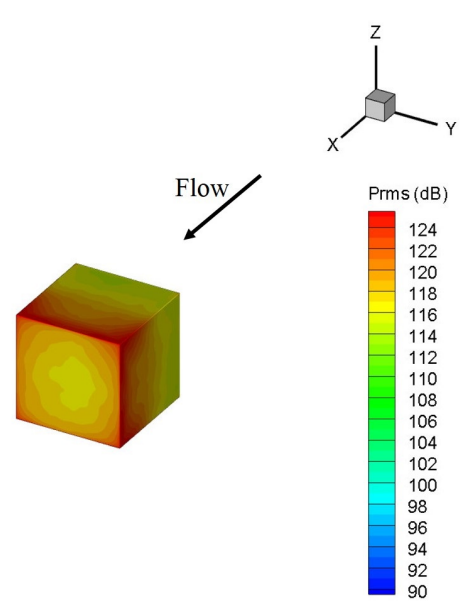

(e)

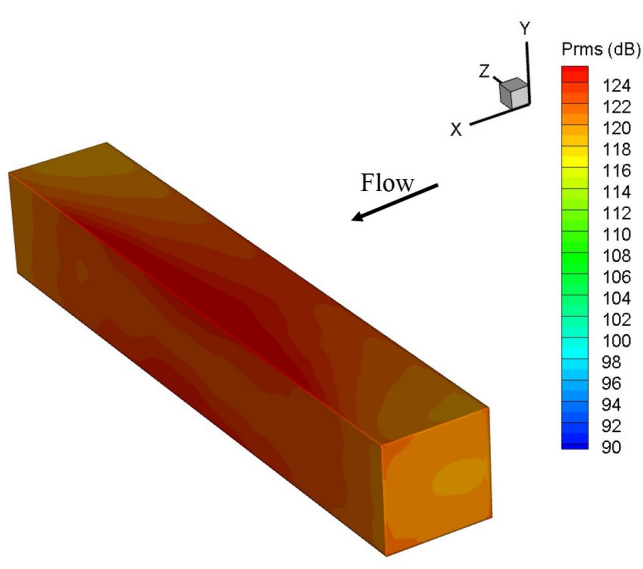

(b)

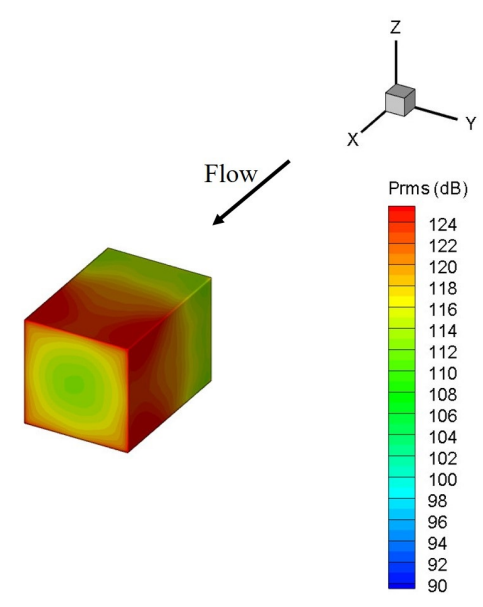

(d)

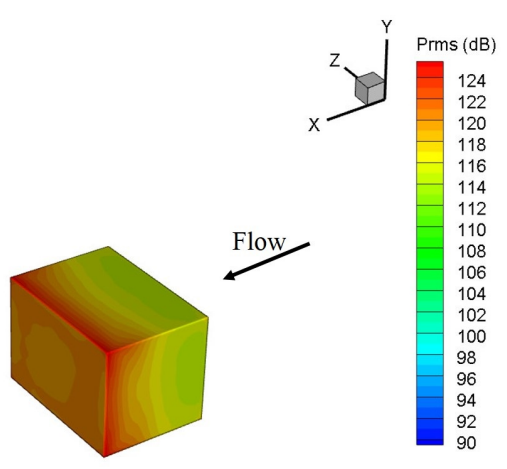

(f)

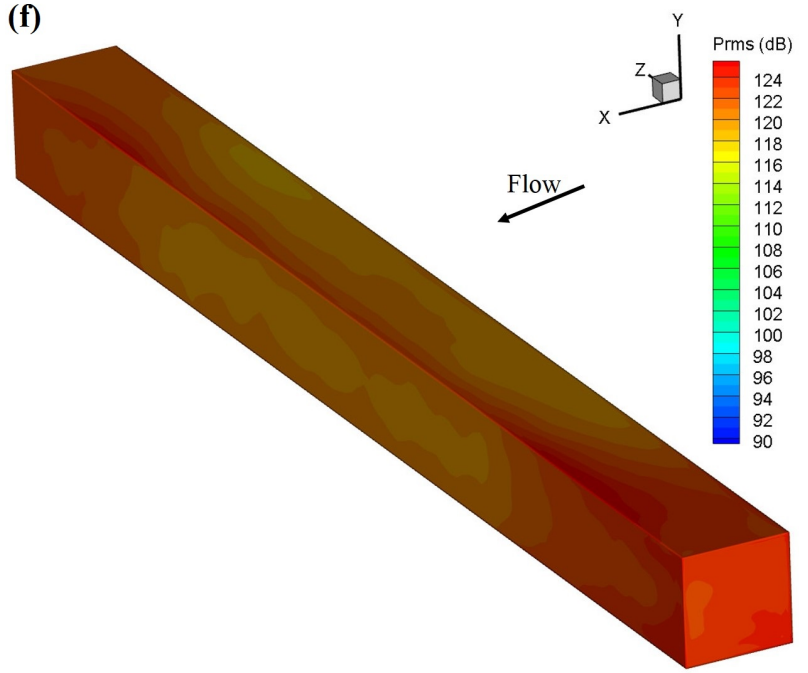

FIG. 16. Contours of the pressure fluctuation for cuboids with different aspect ratios at a Reynolds number of 100,000. (a) $W / L=1 / 6$, (b) $W / L=1 / \sqrt{2}$, (c) $W / L=1$, (d) $W / L=\sqrt{2}$, (e) $W / L=6$, (f) $W / L=12$ 
The near-wall flow patterns obtained from the first layer grids on the top surface of cuboids with representative aspect ratios are shown in Fig. 17. These are coloured by the pressure fluctuations on the top surface. The Nodes are also labeled in Fig. 17 to characterize the near-wall flow topology; explanations of the significance of this critical point can be found in ref. 16. The white lines represent locations of zero mean streamwise velocity. As can be seen from Figs $17(\mathrm{a})$ and $17(\mathrm{~b})$ the largest pressure fluctuations for $W / L<1$ happen at the Nodes labelled 'N1' and 'N2' on the reattachment line. These Nodes move closer to the trailing edge with the increase of the aspect ratio. Since the near-wall flow patterns and distributions of the pressure fluctuations for $W / L=1, \sqrt{2}, 2$ and 3 are similar, only those for $W / L=\sqrt{2}$ are shown in Fig. 17(c). The largest pressure fluctuations are found near Nodes 'N3' and 'N4' close to the rear corners, formed due to the secondary vortex seen in Fig. 9(c). Fig. 17(d)] shows the flow patterns for $W / L=6$, which are similar to those for $W / L=12$, despite flatter streamlines near the rear corner. A Node 'N5' is formed close to the centreline of the top surface $(z / H=0)$ and the pressure fluctuations in the centre are largest.
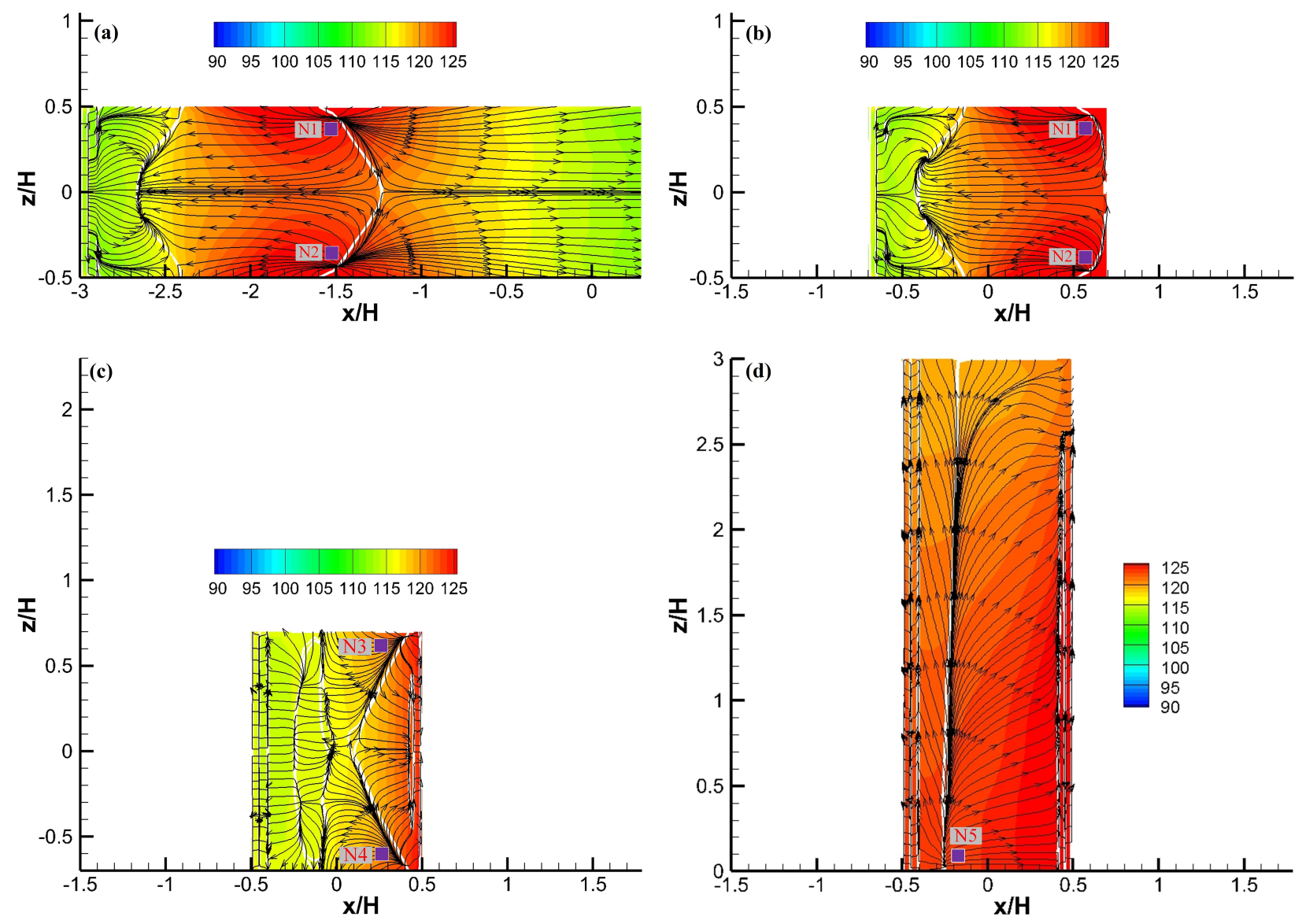

FIG. 17. Mean streamlines and contours of the pressure fluctuation $\left(L_{p}\right)$ on the top surface of cuboids with different aspect ratios at $R e=100,000$ (square: Node). (a) $W / L=1 / 6$, (b) $W / L=1 / \sqrt{2}$, (c) $W / L=\sqrt{2}$, (d) $W / L=6$

\section{EFFECT OF THE ASPECT RATIO ON THE RADIATED NOISE}

Based on the fluctuating pressure on the surfaces of the cuboid obtained from CFD, the far-field noise at receivers $10 \mathrm{~m}(133 H)$ away from the cuboid centre in the streamwise, cross-flow and spanwise directions is predicted using 
the FW-H method. The OASPL at each receiver is calculated by integrating the PSD of the emitted noise up to a Strouhal number $\left(S t=f H / U_{\infty}\right)$ of 4 . Moreover, the predicted noise for each case is then rescaled to correspond to a total surface area of $6 \mathrm{H}^{2}$ corresponding to the cube, namely taking into account a correction:

$$
\begin{aligned}
L_{\text {cor }} & =-10 \log _{10}\left((2 H L+2 H W+2 W L) / 6 H^{2}\right) \\
& = \begin{cases}-10 \log _{10}(1 / 3+2 L / 3 W) & W / L \leq 1 \\
-10 \log _{10}(1 / 3+2 W / 3 L) & W / L>1\end{cases}
\end{aligned}
$$

as illustrated in Fig. 18(a), The predicted OASPL for all the studied cases at the Reynolds number of 100,000 is plotted in Fig. 18(b), As expected, when the aspect ratio is less than 1, the sound along the cross-flow direction at (0, $10,0)$ is very similar to that observed in the spanwise direction at $(0,0,10)$. However, for $W / L>1$, the noise at $(0$, $0,10)$ drops quickly with the increase of the aspect ratio and the reduction rate is faster than that due to the surface area correction shown in Fig. 18(a). For $W / L=12$, the OASPL in the spanwise direction is about $27 \mathrm{~dB}$ less than that in the cross-flow direction. In addition, the sound pressure level in the cross-flow direction at the receiver $(0,10$, $0)$ is always greater than that observed in the streamwise direction at $(10,0,0)$; the greatest difference is about $9 \mathrm{~dB}$, observed for an aspect ratio of 6 .
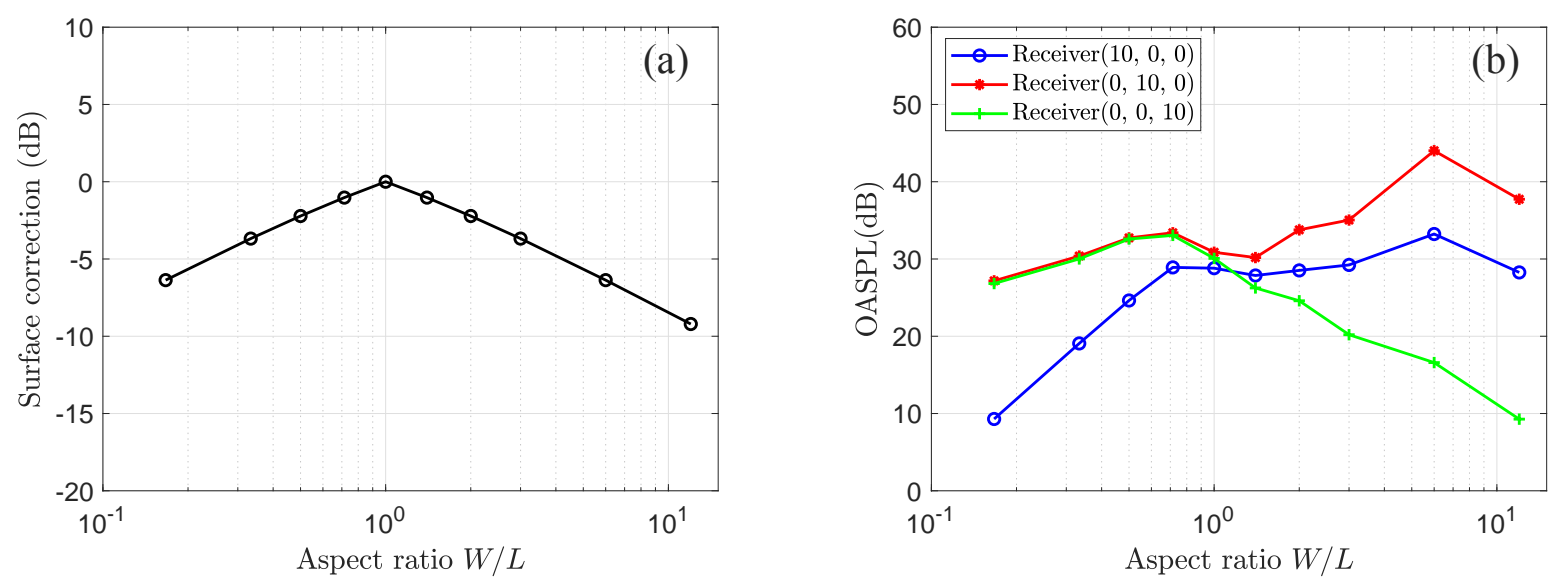

FIG. 18. (a) Surface correction of the OASPL (b) Predicted OASPL at the Reynolds number of 100,000 for cuboids with different aspect ratios ranging from $1 / 6$ to 12

Noise spectra for representative cases at $R e=100,000$ predicted at the three receivers are shown in Fig. 19. The surface area correction (Equation (11) is not implemented for these noise spectra. Since the noise spectra for cases with $W / L<1$ are similar, only the spectra for the bounding cases of $W / L=1 / 6$ and $1 / \sqrt{2}$ are plotted in Fig. 19 . In addition, the main features of the noise spectra for cases with $W / L=2$ and 3 are similar to that observed in the case $W / L=6$, so they are also not shown. As shown in Fig. 19(a) no evident tonal peaks are found in spectra for the receiver in the streamwise direction for any investigated case and, with the increase of the aspect ratio, the noise at Strouhal numbers smaller than 1 gradually increases.

In Fig. 19(b) for the receiver along the cross-flow direction, although no tonal frequency is recognisable for $W / L<1$, a dominant peak centred at about $S t=0.1$ can be clearly seen in the spectra for $W / L=1$ to 12 . For comparison, 

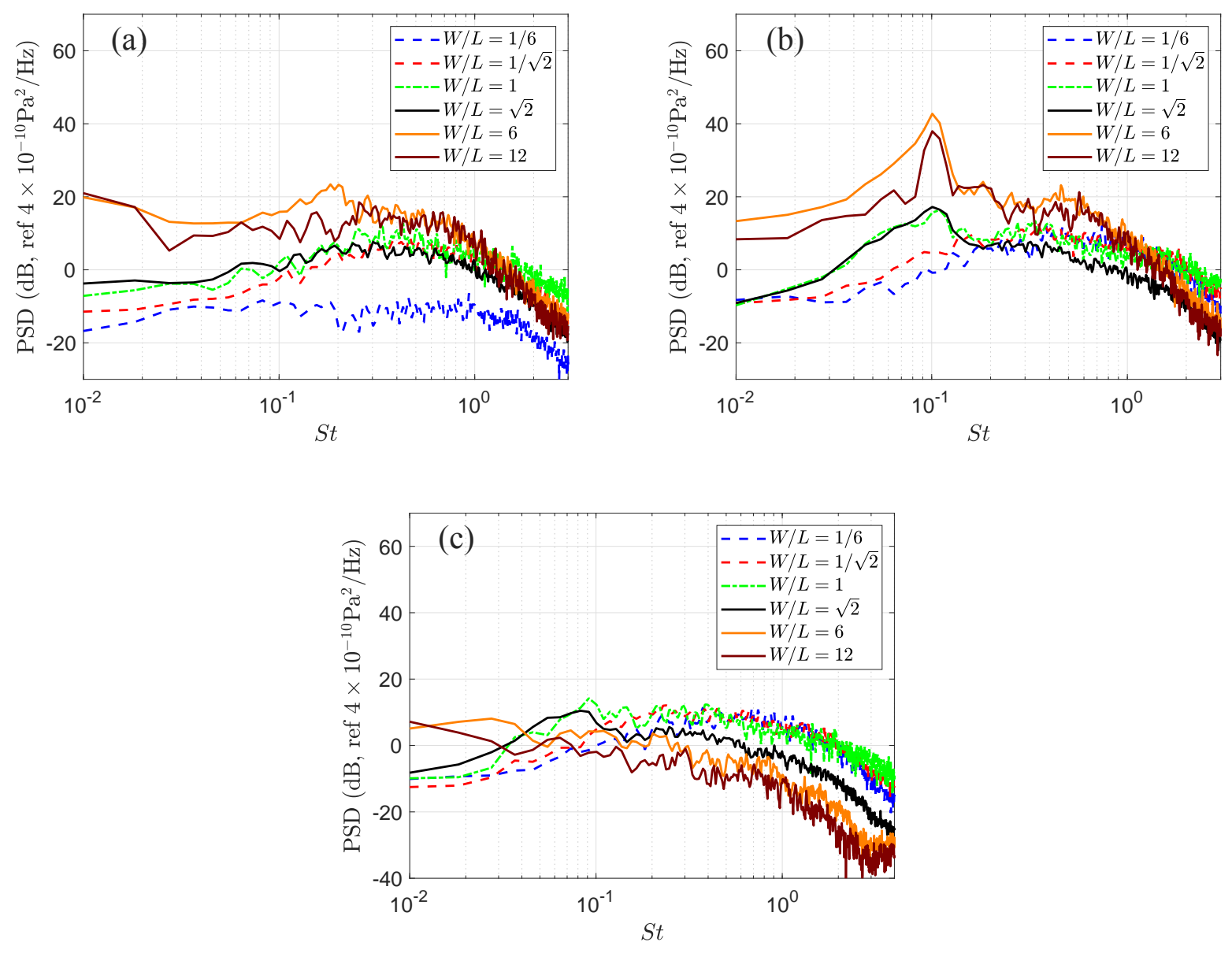

FIG. 19. Noise spectra along the streamwise, cross-flow and spanwise directions respectively for cuboids with different aspect ratios at $R e=100,000$. (a) Receiver $(10,0,0)$, (b) Receiver $(0,10,0)$, (c) Receiver (0, 0, 10)

the peak $S t$ for an infinite square cylinder is about 0.13 .49 The Strouhal number of 0.1 found in these results is consistent with that reported by Porteous et al 50 for square wall-mounted cylinders in the regime $2<W / L<10$. This tonal peak is formed due to the periodic vortex shedding in the wake of the cuboid similar to that observed in the wake of an infinite cylinder (see Fig. 7). With the increase of the aspect ratio, the magnitude of this Aeolian tone keeps increasing while the peak becomes more and more narrow-band. Whereas the frequencies of the dominant peak changed with respect to the aspect ratio for finite wall-mounted cylinders studied by Porteous et al., 50 the position of the tonal peak for the cuboids with different aspect ratios studied here is quite constant. In addition, although a bifurcation at frequencies lower than the main tonal peak near $S t=0.1$ emerges for a wall-mounted cylinder with $W / L=12.9, \sqrt[50]{5}$ this is not observed in the spectrum for $W / L=12$ in Fig. 19(b). This suggests that the dual cellular shedding modes for a finite wall-mounted cylinder with the aspect ratio close to 12 do not happen for cuboids in free space, where only one shedding mode is identified in the current numerical work. From Fig. 19(c), for the receiver in the spanwise direction, a broadband peak centred at about $S t=0.1$ can be seen in the case with $W / L=1$. However, when the aspect ratio exceeds 1 , this broadband peak disappears.

The predicted noise in the streamwise, cross-flow and spanwise directions at different Reynolds numbers for all the studied values of aspect ratio is summarized in Fig. 20. The OASPL is corrected for the surface area according to 
Equation (11) and is rescaled by $U_{\infty}^{6}$ to correspond to $40 \mathrm{~m} / \mathrm{s}(R e=200,000)$. This means that for the incoming flow of $10 \mathrm{~m} / \mathrm{s}(R e=50,000) 36 \mathrm{~dB}$ is added, while that for $U_{\infty}=20 \mathrm{~m} / \mathrm{s}(R e=100,000) 18 \mathrm{~dB}$ is added.
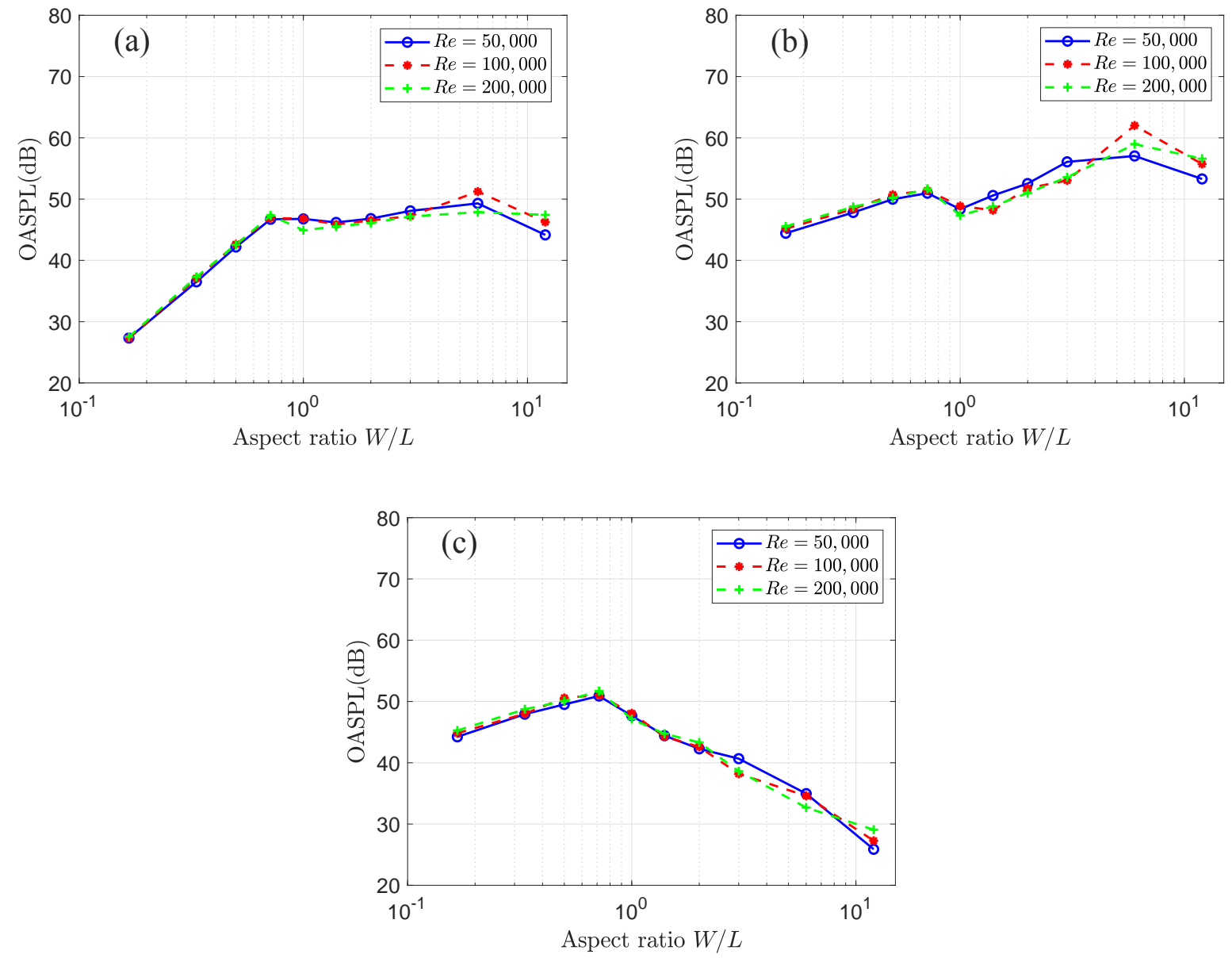

FIG. 20. A summary of the predicted OASPL along the streamwise, cross-flow and spanwise directions respectively. (a) Noise at the receiver $(10,0,0),(b)$ Noise at the receiver $(0,10,0),(\mathrm{c})$ Noise at the receiver $(0,0,10)$

As shown in Fig. 20, the trends of the OASPL with respect to the aspect ratio are similar at the different Reynolds numbers for all three directions, especially for cases with small values of $W / L$. This confirms that the emitted noise follows a sixth power law with respect to the flow speed. For all three directions shown in Fig. 20, the maximum OASPL for $W / L \leq 1$ is always observed for the case with $W / L=1 / \sqrt{2}$. In this case, the separated flow from the leading edge reattaches close to the trailing edge. For $W / L>1$, in general, the noise along the streamwise direction shown in Fig. 20(a) changes only slightly with increase of the aspect ratio, while the noise in the cross-flow direction displayed in Fig. 20(b) keeps growing and the sound along the spanwise direction in Fig. 20(c) decreases rapidly.

\section{CONCLUSIONS}

The effect of aspect ratio on the flow past cuboids and the emitted noise has been investigated numerically. Ten aspect ratios from 1/6 to 12 are taken into account at $R e=50,000,100,000$ and 200,000.

The main mean flow features change only slightly with variations of the Reynolds number; however they are strongly 
influenced by the aspect ratio. The separated flow reattaches to the top and lateral surfaces of cuboids with aspect ratios smaller than 1. In particular, it reattaches at the trailing edge for $W / L=1 / \sqrt{2}$. However, for further increases of aspect ratio, the separated flow convects downstream to the wake instead of reattaching to the top surface and a secondary vortex is formed near the trailing edge in the $z=0$ plane. The lowest mean drag coefficient is observed for $W / L=1 / \sqrt{2}$ and the trend of the mean drag coefficient with respect to the aspect ratio is the opposite to that of the base pressure coefficient. The largest pressure fluctuations occur in the region of the flow reattachment for $W / L<1$ while they occur close to the trailing edge for $W / L \geq 1$ due to the formation of the secondary vortex.

No evident tonal peaks can be observed in the noise spectra for cuboids with $W / L \leq 1$ at receivers in the streamwise, cross-flow or spanwise directions. For aspect ratios greater than 1, a dominant tonal peak centred at about $S t=0.1$ is found in the noise spectra along the cross-flow direction. With the increase of the aspect ratio, the magnitude of this dominant peak gradually increases but the position of the central frequency remains constant, which is different from the noise spectra of finite wall-mounted cylinders with similar aspect ratios presented in the literature. In addition, the far-field noise from cuboids with different aspect ratios grows at a rate close to $60 \log _{10} U_{\infty}$ with respect to the incoming flow speed.

\section{ACKNOWLEDGMENTS}

This research was supported by the University of Southampton and China Scholarship Council (CSC). The simulations in this paper were carried out on the iridis 4 supercomputer at the University of Southampton. All data published in this paper are openly available from the University of Southampton repository at https://doi.org/10.5258/SOTON/D1214

${ }^{1}$ B. J. Vickery, "Fluctuating lift and drag on a long cylinder of square cross-section in a smooth and in a turbulent stream," Journal of Fluid Mechanics 25, 481-494 (1966).

${ }^{2}$ D. Lyn, S. Einav, W. Rodi, and J.-H. Park, "A laser-doppler velocimetry study of ensemble-averaged characteristics of the turbulent near wake of a square cylinder," Journal of Fluid Mechanics 304, 285-319 (1995).

${ }^{3}$ J. Shang, Q. Zhou, M. M. Alam, H. Liao, and S. Cao, "Numerical studies of the flow structure and aerodynamic forces on two tandem square cylinders with different chamfered-corner ratios," Physics of Fluids 31, 075102 (2019).

${ }^{4}$ H. Bai and M. M. Alam, "Dependence of square cylinder wake on Reynolds number," Physics of Fluids 30, 015102 (2018).

${ }^{5}$ H. Jiang and L. Cheng, "Hydrodynamic characteristics of flow past a square cylinder at moderate Reynolds numbers," Physics of Fluids 30, 104107 (2018).

${ }^{6}$ A. Okajima, "Strouhal numbers of rectangular cylinders," Journal of Fluid Mechanics 123, 379-398 (1982).

${ }^{7}$ H. Wang, Y. Zhou, C. Chan, and K. Lam, "Effect of initial conditions on interaction between a boundary layer and a wall-mounted finite-length-cylinder wake," Physics of Fluids 18, 065106 (2006).

${ }^{8}$ H. Wang and Y. Zhou, "The finite-length square cylinder near wake," Journal of Fluid Mechanics 638, $453-490$ (2009).

${ }^{9}$ L. Zou, S. Liao, C. Liu, Y. Wang, and Z. Zhai, "Aspect ratio effect on shock-accelerated elliptic gas cylinders," Physics of Fluids 28, 036101 (2016).

${ }^{10}$ P. Kumar, N. P. Manelil, and S. Tiwari, "Effects of shear intensity and aspect ratio on three-dimensional wake characteristics of flow past surface mounted circular cylinder," Physics of Fluids 31, 043602 (2019).

${ }^{11}$ R. Porteous, D. J. Moreau, and C. J. Doolan, "A review of flow-induced noise from finite wall-mounted cylinders," Journal of Fluids and Structures 51, 240-254 (2014).

${ }^{12}$ Y. Yauwenas, R. Porteous, D. J. Moreau, and C. J. Doolan, "The effect of aspect ratio on the wake structure of finite wall-mounted square cylinders," Journal of Fluid Mechanics 875, 929-960 (2019). 
${ }^{13}$ D. Zhang, L. Cheng, H. An, and M. Zhao, "Direct numerical simulation of flow around a surface-mounted finite square cylinder at low Reynolds numbers," Physics of Fluids 29, 045101 (2017).

${ }^{14}$ S. Behera and A. Saha, "Characteristics of the flow past a wall-mounted finite-length square cylinder at low Reynolds number with varying boundary layer thickness," Journal of Fluids Engineering 141, 061204 (2019).

${ }^{15}$ Y. Cao, T. Tamura, and H. Kawai, "Investigation of wall pressures and surface flow patterns on a wall-mounted square cylinder using very high-resolution cartesian mesh," Journal of Wind Engineering and Industrial Aerodynamics 188, 1-18 (2019).

${ }^{16}$ J. M. Délery, "Robert Legendre and Henri Werlé: toward the elucidation of three-dimensional separation," Annual Review of Fluid Mechanics 33, 129-154 (2001).

${ }^{17}$ A. Beitel, H. Heng, and D. Sumner, "The effect of aspect ratio on the aerodynamic forces and bending moment for a surface-mounted finite-height cylinder," Journal of Wind Engineering and Industrial Aerodynamics 186, 204-213 (2019).

${ }^{18}$ A. Š. Glumac, H. Hemida, and R. Höffer, "Wind energy potential above a high-rise building influenced by neighboring buildings: An experimental investigation," Journal of Wind Engineering and Industrial Aerodynamics 175, 32-42 (2018).

${ }^{19}$ D. J. Moreau and C. J. Doolan, "Flow-induced sound of wall-mounted finite length cylinders," AIAA journal 51, $2493-2502$ (2013).

${ }^{20}$ R. Porteous, D. J. Moreau, and C. J. Doolan, "The aeroacoustics of finite wall-mounted square cylinders," Journal of Fluid Mechanics 832, 287-328 (2017).

${ }^{21}$ S. Becker, C. Hahn, M. Kaltenbacher, and R. Lerch, "Flow-induced sound of wall-mounted cylinders with different geometries," AIAA journal 46, 2265-2281 (2008).

${ }^{22}$ B. Lee, "The effect of turbulence on the surface pressure field of a square prism," Journal of Fluid Mechanics 69, 263-282 (1975).

${ }^{23}$ W. King and E. Pfizenmaier, "An experimental study of sound generated by flows around cylinders of different cross-section," Journal of Sound and Vibration 328, 318-337 (2009).

${ }^{24}$ D. K. Kaushik, D. E. Keyes, and B. F. Smith, "Newton-Krylov-Schwarz methods for aerodynamics problems: compressible and incompressible flows on unstructured grids." Tech. Rep. (Argonne National Lab., IL (US), 1999).

${ }^{25}$ S. B. Pope, Turbulent flows (Cambridge Univ. Press, 2001).

${ }^{26}$ D. C. Wilcox et al., Turbulence modeling for CFD (second edition, DCW Industries La Canada, CA, 1998).

${ }^{27}$ A. N. Kolmogorov, "The local structure of turbulence in incompressible viscous fluid for very large reynolds numbers," Dokl. Akad. Nauk SSSR 30, 301-305 (1941).

${ }^{28}$ J. Smagorinsky, "General circulation experiments with the primitive equations: I. the basic experiment," Monthly Weather Review 91, 99-164 (1963).

${ }^{29}$ D. K. Lilly, On the application of the eddy viscosity concept in the inertial sub-range of turbulence (National Center for Atmospheric Research, 1966).

${ }^{30}$ M. Germano, U. Piomelli, P. Moin, and W. H. Cabot, "A dynamic subgrid-scale eddy viscosity model," Physics of Fluids A: Fluid Dynamics (1989-1993) 3, 1760-1765 (1991).

${ }^{31}$ P. Moin and K. Mahesh, "Direct numerical simulation: a tool in turbulence research," Annual Review of Fluid Mechanics 30, 539-578 (1998).

${ }^{32}$ P. R. Spalart and S. R. Allmaras, "A one equation turbulence model for aerodynamic flows," AIAA Journal 94, 439 (1992).

${ }^{33}$ P. Spalart, W. Jou, M. Strelets, S. Allmaras, et al., "Comments on the feasibility of LES for wings, and on a hybrid RANS/LES approach," Advances in DNS/LES 1, 4-8 (1997).

${ }^{34}$ J. Fröhlich and D. von Terzi, "Hybrid LES/RANS methods for the simulation of turbulent flows," Progress in Aerospace Sciences 44, 349-377 (2008).

${ }^{35}$ F. Menter and M. Kuntz, "Adaptation of eddy-viscosity turbulence models to unsteady separated flow behind vehicles," in The Aerodynamics of Heavy Vehicles: Trucks, Buses, and Trains (Springer, 2004) pp. 339-352.

${ }^{36}$ P. R. Spalart, S. Deck, M. Shur, K. Squires, M. K. Strelets, and A. Travin, "A new version of detached-eddy simulation, resistant to ambiguous grid densities," Theoretical and Computational Fluid Dynamics 20, 181-195 (2006).

${ }^{37}$ M. L. Shur, P. R. Spalart, M. K. Strelets, and A. K. Travin, "A hybrid rans-les approach with delayed-des and wall-modelled les capabilities," International Journal of Heat and Fluid Flow 29, 1638-1649 (2008).

${ }^{38}$ S. R. Allmaras and F. T. Johnson, "Modifications and clarifications for the implementation of the spalart-allmaras turbulence model," in Seventh international conference on computational fluid dynamics (ICCFD7) (2012) pp. 1-11. 
${ }^{39}$ M. Shur, P. Spalart, M. Strelets, and A. Travin, "Detached-eddy simulation of an airfoil at high angle of attack," in Engineering turbulence modelling and experiments 4 (Elsevier, 1999) pp. 669-678.

${ }^{40}$ M. J. Lighthill, "On sound generated aerodynamically. i. general theory," Proceedings of the Royal Society of London A: Mathematical, Physical and Engineering Sciences 211, 564-587 (1952).

${ }^{41}$ M. J. Lighthill, "On sound generated aerodynamically. ii. turbulence as a source of sound," Proceedings of the Royal Society of London A: Mathematical, Physical and Engineering Sciences 222, 1-32 (1954).

${ }^{42}$ N. Curle, "The influence of solid boundaries upon aerodynamic sound," Proceedings of the Royal Society of London. Series A. Mathematical and Physical Sciences 231, 505-514 (1955).

${ }^{43}$ J. E. Ffowcs Williams and D. L. Hawkings, "Sound generation by turbulence and surfaces in arbitrary motion," Philosophical Transactions of the Royal Society of London. Series A, Mathematical and Physical Sciences 264, 321-342 (1969).

${ }^{44}$ F. Fahy and D. Thompson, Fundamentals of sound and vibration 2nd edition (CRC Press, 2016).

${ }^{45}$ S. Glegg and W. Devenport, Aeroacoustics of low Mach number flows: fundamentals, analysis, and measurement (Academic Press, 2017).

${ }^{46}$ Y. Wang, D. Thompson, and Z. Hu, "Effect of wall proximity on the flow over a cube and the implications for the noise emitted," Physics of Fluids 31, 077101 (2019).

${ }^{47}$ S. Depardon, J. Lasserre, J. Boueilh, L. Brizzi, and J. Borée, "Skin friction pattern analysis using near-wall PIV," Experiments in Fluids 39, 805-818 (2005).

${ }^{48}$ T. Fox and G. West, "Fluid-induced loading of cantilevered circular cylinders in a low-turbulence uniform flow. part 1: mean loading with aspect ratios in the range 4 to 30," Journal of Fluids and Structures 7, 1-14 (1993).

${ }^{49}$ C. Norberg, "Flow around rectangular cylinders: pressure forces and wake frequencies," Journal of Wind Engineering and Industrial Aerodynamics 49, 187-196 (1993).

${ }^{50}$ R. Porteous, D. J. Moreau, and C. J. Doolan, "The aeroacoustics of finite wall-mounted square cylinders," Journal of Fluid Mechanics 832, 287-328 (2017). 